\title{
Actuator Fault Diagnosis with Application to a Diesel Engine Testbed
}

\author{
Boulaïd Boulkroune, ${ }^{1}$ Abdel Aitouche, ${ }^{1}$ Vincent Cocquempot, ${ }^{2}$ \\ Li Cheng, ${ }^{3}$ and Zhijun Peng ${ }^{3}$ \\ ${ }^{1}$ Hautes Etudes d'Ingénieur, Centre de Recherche en Informatique, Signal et Automatique de Lille (CRIStAL), \\ 13 rue de Toul, 59046 Lille, France \\ ${ }^{2}$ Lille 1 University, Centre de Recherche en Informatique, Signal et Automatique de Lille (CRIStAL), \\ rue Paul Langevin, 59655 Villeneuve d'Ascq, France \\ ${ }^{3}$ Department of Engineering \& Design, University of Sussex, Room 2D3, Richmond Building, Brighton BN1 9QT, UK
}

Correspondence should be addressed to Boulaïd Boulkroune; boulkrb@gmail.com

Received 3 September 2014; Revised 20 February 2015; Accepted 26 February 2015

Academic Editor: Kui Fu Chen

Copyright (C) 2015 Boulaïd Boulkroune et al. This is an open access article distributed under the Creative Commons Attribution License, which permits unrestricted use, distribution, and reproduction in any medium, provided the original work is properly cited.

\begin{abstract}
This work addresses the issues of actuator fault detection and isolation for diesel engines. We are particularly interested in faults affecting the exhaust gas recirculation (EGR) and the variable geometry turbocharger (VGT) actuator valves. A bank of observerbased residuals is designed using a nonlinear mean value model of diesel engines. Each residual on the proposed scheme is based on a nonlinear unknown input observer and designed to be insensitive to only one fault. By using this scheme, each actuator fault can be easily isolated since only one residual goes to zero while the others do not. A decision algorithm based on multi-CUSUM is used. The performances of the proposed approach are shown through a real application to a Caterpillar 3126b engine.
\end{abstract}

\section{Introduction}

On-board diagnosis of automotive engines has become increasingly important because of environmentally based legislative regulations such as OBDII (On-Board DiagnosticsII) [1]. On-board diagnosis is also needed to guarantee highperformance engine behavior. Today, due to the legislation, the majority of the code in modern engine management systems is dedicated to diagnosis.

Model-based diagnosis of automotive engines has been considered in earlier papers (see, e.g., $[2,3]$ ), to name only a few. However, the engines investigated in these previous works were all gasoline-fuelled and did not include exhaust gas recirculation (EGR) and variable geometry turbocharger (VGT). Both of these components make the diagnosis problem significantly more difficult since the air flows through the EGR valve, and also the exhaust side of the engine has to be taken into account. An interesting approach to modelbased air-path faults detection for an engine which includes EGR and VGT can be found in $[4,5]$. By using several models in parallel, where each one is sensitive to one kind of fault, predicted outputs are compared and a diagnosis is provided. The hypothesis test methodology proposed in [4] deals with the multifault detection in air-path system. In [5] the authors propose an extended adaptive Kalman filter to find which faulty model best matches with measured data; then a structured hypothesis allows going back to the faults. A structural analysis for air path of an automotive diesel engine was developed in order to study the monitorability of the system [6-8]. Other approaches to detect intake leakages in diesel engines based on adaptive observers are proposed in $[9,10]$ and recently in [11]. Note that, in all these approaches, the leakage size is assumed to be constant. To overcome this limitation, an approach based on a nonlinear unknown input observer (NIUO), for intake leakage detection, is proposed in [12]. No a priori assumption about the leakage size is made. In $[13,14]$, an interesting method for bias compensation in model-based estimation using model augmentation is proposed. The extended Kalman filter (EKF) is used for estimating the states of the augmented model. Also, 


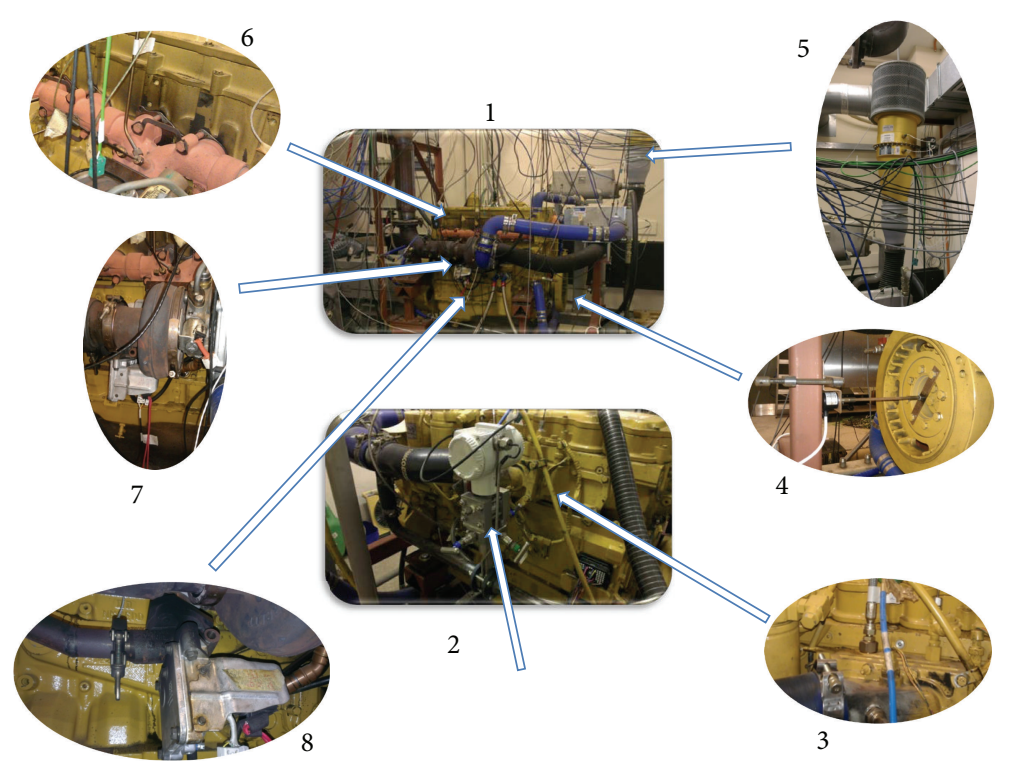

FIgURE 1: Caterpillar testbed 3126, Sussex University.

the observability of the augmented model is well discussed. Recently, an automated model-based and data-driven design methodology for automotive engine fault detection and isolation (FDI) is proposed in [15]. This methodology, which combines model-based sequential residual generation and data-driven statistical residual evaluation, is used to create a complete FDI system for an automotive diesel engine.

The problem of designing unknown input observers (UIO) has received great attention in literature. This problem is motivated by certain applications such as fault diagnosis and control system design. If this issue in linear case is well solved, it remains an open problem in the nonlinear case. The first unknown input observers dedicated to linear systems were proposed in $[16,17]$. Necessary and sufficient conditions of the existence of the UIO have been well established. New sufficient conditions formulated in terms of linear matrix inequality (LMI) were given by [18]. This result is extended in [12] to cover a wide class of nonlinear systems that cannot be treated by the previous approach as well as nonlinear systems with a large Lipschitz constant.

Our aim is to address the issue of actuator fault detection and isolation for diesel engines. Indeed, actuators (EGR and VGT) fault diagnosis is necessary and crucial to guarantee its healthy operation. In this work, a fault detection and isolation (FDI) system is developed. The proposed FDI system is composed of two parts: residual generation and decision system. A multiobserver strategy is used for residual generation. A mean model of diesel engine is exploited for the design of a set of nonlinear unknown input observers. These observers are designed in order to estimate the states behavior without any knowledge of the unknown inputs. The sufficient conditions of the existence of the NUIO are given in terms of linear matrix inequalities (LMIs). The advantage of this method is that no a priori assumption on the unknown input is required and also can be employed for a wider class of nonlinear systems. To achieve fault detection and isolation, a decision system based on a statistical approach, multiCUSUM (cumulative sum), is used to process the resulting set of residuals.

This paper is organized as follows. The experimental setup is described in Section 2. The diesel engine model and its validation are described in Section 3. A nonlinear unknown input observer is presented in Section 4. Section 5 describes the residual generation system while the decision system is presented in Section 6. The experimental results and discussion are presented in Section 7. Finally, conclusion and future works are given in the last section.

The notations used in this paper are quite standard. Let $\mathfrak{R}$ denote the set of real numbers. The set of $p$ by $q$ real matrices is denoted as $\Re^{p \times q}$. $A^{T}$ and $A^{-1}$ represent the transpose of $A$ and its left inverse (assuming $A$ has full column rank), respectively. $I_{r}$ represents the identity matrix of dimension $r$. $(*)$ is used for the blocks induced by symmetry. $\|\cdot\|$ represents the usual Euclidean norm. $\boldsymbol{\Omega}_{2}^{r}$ denotes the Lebesgue space. $f_{a, i}$ denotes the $i$ th component of the vector $f_{a}$.

\section{Experimental Installation}

The testbed is built with a Caterpillar 3126b truck engine coupled to a SCHORCH dynamometer controlled by $\mathrm{CP}$ Cadet Software. The Caterpillar engine is presented in Figure 1. The front and back view of the testbed are shown by the pictures numbered (1) and (2), respectively. Engine's components are inlet manifold (3), encoder for measuring of the engine speed (4), intake air flow meter (5), exhaust manifold (6), GT3782VA variable geometry turbocharger, (7) and exhaust gas valve (8). In order to enable a transient control of the EGR and VGT, a dSPACE MicroAutoBox 1401/1501 real-time controller is connected (see Figure 2). Apart from the standard OEM electronic sensors built in for ECU and dynamometer control, additional sensors and actuators have 


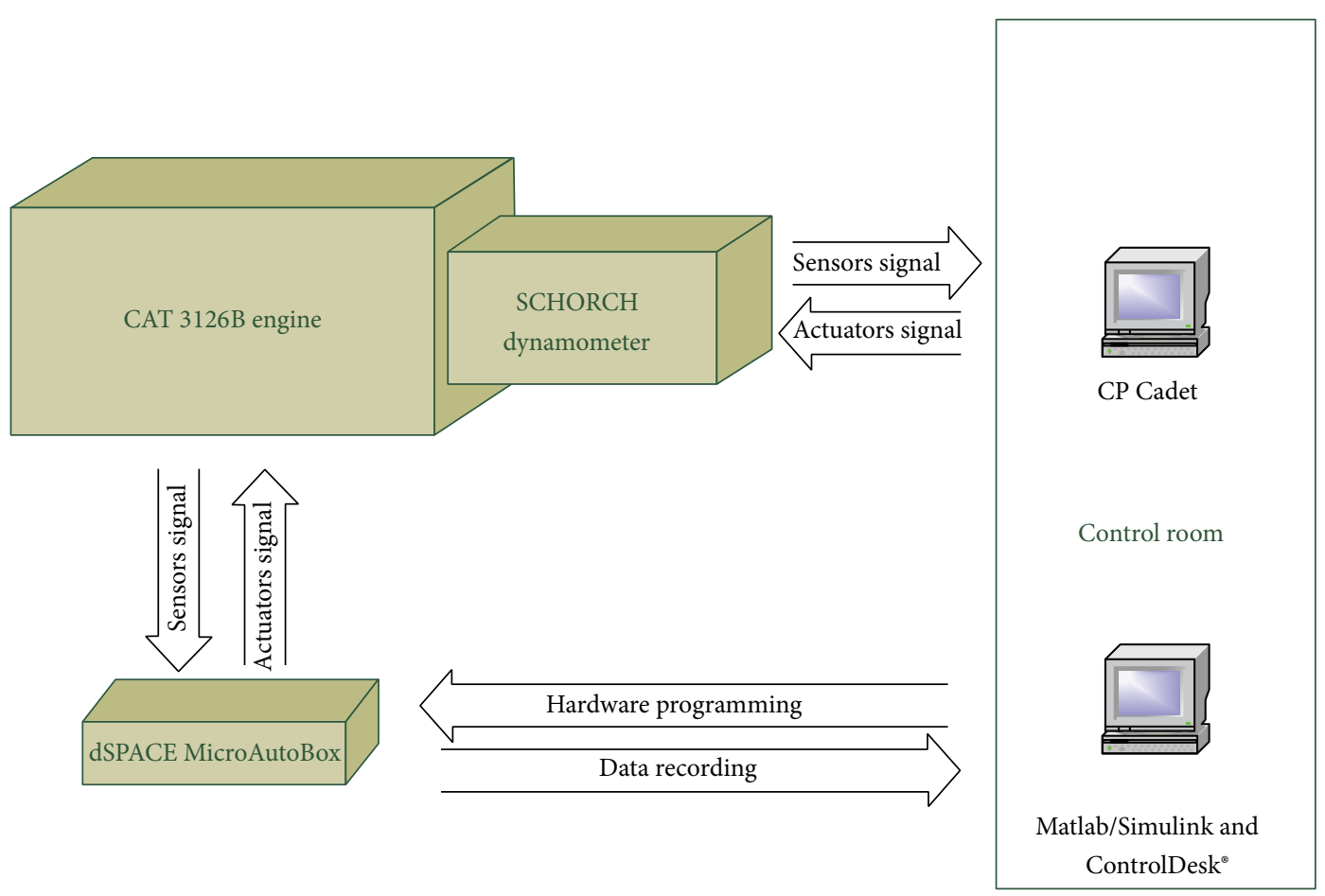

FIGURE 2: Experimental installation schematic, Sussex University.

TABLE 1: Sensors and actuators list.

\begin{tabular}{ll}
\hline Sensors & Actuators \\
\hline Inlet temperature sensor & $\begin{array}{l}\text { EGR valve drive actuator } \\
\text { VGT vanes drive } \\
\text { actuator }\end{array}$ \\
Inlet air flow meter & \\
Inlet pressure sensor & \\
Pre-turbo exhaust pressure sensor & \\
Acceleration pedal position sensor & \\
Engine speed sensor & \\
Inlet manifold oxygen sensor & \\
Exhaust manifold oxygen sensor & \\
Exhaust opacity sensor (AVL & \\
Opacimeter 439) & \\
Exhaust emission sensor (Testo 350 & \\
Engine test kit) & \\
EGR position feedback sensor & \\
VGT position feedback sensor &
\end{tabular}

been wired in specifically for the MicroAutoBox. These sensors and actuators are listed in Table 1.

The data and control signal flow are illustrated in Figure 2. The engine is connected with two control platforms, which are CP Cadet and dSPACE Control Desk. The engine tests are conducted and monitored by CP Cadet Platform while the EGR and VGT valve positions can be adjusted through dSPACE Control Desk in real time. Testing data can be collected from both platforms and used for data analysis purpose.

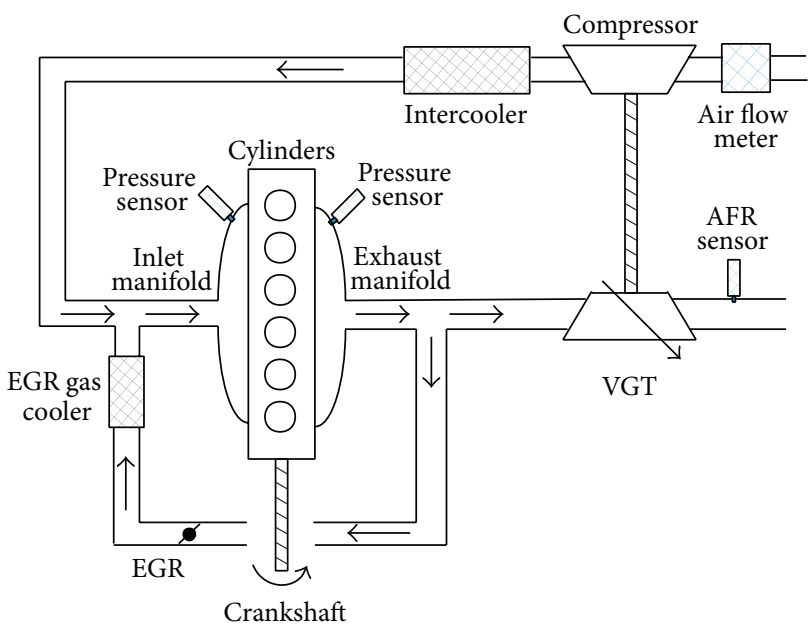

FIGURE 3: Turbocharged air-intake system schematic.

The specification of the Caterpillar 3126b midrange truck engine is given in Table 3 .

\section{Engine Model and Validation}

The considered diesel engine is a six-cylinder engine with a high-pressure EGR and VGT. A principle illustration scheme of the air-path system is shown in Figure 3. It consists of two parts: the turbocharger and exhaust gas recirculation. The turbocharger is a turbine driven by the exhaust gas and connected via a common shaft to the compressor, which 
compresses the air in the intake. The exhaust gas recirculation (EGR) allows for recirculating gas from the exhaust manifold to the intake manifold. First, the mixture of air coming from the compressor and exhaust gas coming through the EGR valve enters the intake manifold before injecting it into the cylinders. Then, the fuel is injected directly in the cylinders and burned, producing the torque on the crank shaft. Exhaust gases are expelled into the exhaust manifold. As shown in Figure 3, part of exhaust gas comes out from the exhaust manifold through the turbine and the other part is recirculated through EGR valve. We noted that the temperature of gases (compressed air and EGR gas) entering the intake manifold is reduced using the intercooler and the EGR cooler.

The mean value engine modeling approach is one of the most considered approaches in the literature [19]. It uses temporal and spatial averages of relevant temperatures, pressures, and mass flow rates. The engine model is derived based on the laws of conservation of mass and energy and also on the ideal gas law (see, for instance, $[5,20]$ ). As an example, the pressure dynamics in the inlet manifold is obtained by differentiating the ideal gas law equation $P V=m R T$. The complete considered mean value model is expressed as follows [5]:

$$
\begin{gathered}
\dot{P}_{\text {Inlet }} \\
=\frac{1}{V_{\text {Inlet }}}\left(\frac{R_{\text {Air }} c_{p, \text { Air }}}{c_{v, \text { Air }}} W_{\mathrm{HFM}} T_{\mathrm{CAC}}+\frac{R_{\mathrm{Exh}} c_{p, \text { Exh }}}{c_{v, \mathrm{Exh}}} W_{\mathrm{EGR}} T_{\mathrm{EGR}}\right. \\
\left.-\frac{R_{\text {Inlet }} c_{p \text {,Inlet }}}{c_{v, \text { Inlet }}} W_{\text {Inlet }} T_{\text {Inlet }}\right) \\
\dot{m}_{\text {Air }}=W_{\mathrm{HFM}}-\frac{m_{\mathrm{Air}}}{m_{\mathrm{Air}}+m_{\mathrm{EGR}}} W_{\text {Inlet }} \\
\dot{m}_{\mathrm{EGR}}=W_{\mathrm{EGR}}-\frac{m_{\mathrm{EGR}}}{m_{\mathrm{Air}}+m_{\mathrm{EGR}}} W_{\text {Inlet }}, \\
\dot{m}_{\mathrm{Exh}}=W_{\mathrm{Exh}}-W_{\mathrm{Turb}}-W_{\mathrm{EGR}}
\end{gathered}
$$

with

$$
\begin{aligned}
\Psi\left(\frac{p_{1}}{p_{0}}\right)= & \left\{\begin{array}{l}
\sqrt{\frac{2 \kappa}{\kappa-1}\left\{\left(\left(\frac{p_{1}}{p_{0}}\right)^{2 / \kappa}-\left(\frac{p_{1}}{p_{0}}\right)^{(\kappa+1) / \kappa}\right)\right\}} \\
\left.\sqrt{\kappa\left(\frac{2}{\kappa+1}\right)^{(\kappa+1) /(\kappa-1)}} \frac{p_{1}}{p_{0}}\right) \geq\left(\frac{2}{\kappa+1}\right)^{\kappa /(\kappa-1)}
\end{array}\right. \\
& W_{\mathrm{EGR}}=\frac{A_{\mathrm{EGR}} P_{\text {Exh }}}{\sqrt{R_{\text {Exh }} T_{\text {Exh }}} \Psi_{\kappa_{\mathrm{Exh}}}\left(\frac{P_{\text {Inlet }}}{P_{\text {Exh }}}\right),} \\
W_{\text {Inlet }}= & f_{\text {vol }}\left(N_{\text {Eng }}, \frac{P_{\text {Inlet }}}{T_{\text {Inlet }} R_{\text {Inlet }}}\right) \frac{N_{\text {Eng }} P_{\text {Inlet }}}{T_{\text {Inlet }} R_{\text {Inlet }}} \frac{V_{\text {Eng }}}{120}, \\
& T_{\text {Inlet }}=\frac{P_{\text {Inlet }} V_{\text {Inlet }}}{\left(m_{\text {Air }}+m_{\text {EGR }}\right) R_{\text {Inlet }}},
\end{aligned}
$$

$$
\begin{aligned}
& T_{\text {EGR }}=\left(\frac{P_{\text {Inlet }}}{P_{\text {Exh }}}\right)^{\left(\kappa_{\mathrm{Exh}}-1\right) / \kappa_{\mathrm{Exh}}} T_{\mathrm{Exh}}, \\
& W_{\text {Exh }}=W_{\text {Inlet }}+W_{\text {Fuel }} \text {, } \\
& T_{\text {Exh }}=T_{\text {Inlet }}+\frac{Q_{\text {LHV }} h\left(W_{\text {Fuel }}, N_{\text {Eng }}\right)}{c_{p, \text { Exh }}\left(W_{\text {Inlet }}+W_{\text {Fuel }}\right)}, \\
& P_{\mathrm{Exh}}=\frac{m_{\mathrm{Exh}} R_{\mathrm{Exh}} T_{\mathrm{Exh}}}{V_{\mathrm{Exh}}} \text {, } \\
& W_{\text {Turb }}=\frac{P_{\text {Exh }}}{\sqrt{T_{\text {Exh }}}} \tau\left(\frac{P_{\text {Exh }}}{P_{\text {Atm }}}, u_{\mathrm{XVNT}}\right) \text {, } \\
& R_{\text {Inlet }}=\frac{R_{\mathrm{Air}} m_{\mathrm{Air}}+R_{\mathrm{Exh}} m_{\mathrm{EGR}}}{m_{\mathrm{Air}}+m_{\mathrm{EGR}}}, \\
& c_{v, \text { Inlet }}=\frac{c_{v, \mathrm{Air}} m_{\mathrm{Air}}+c_{v, \mathrm{Exh}} m_{\mathrm{EGR}}}{m_{\mathrm{Air}}+m_{\mathrm{EGR}}}, \\
& c_{p \text {,Inlet }}=c_{v \text {,Inlet }}+R_{\text {Inlet }} \text {, } \\
& A_{\mathrm{EGR}}=A_{\mathrm{EGRmax}} f_{\mathrm{EGR}}\left(u_{\mathrm{EGR}}\right) \text {, }
\end{aligned}
$$

where $P_{\text {Inlet }}$ is the pressure in intake manifold. $m_{\text {Air }}$ and $m_{\mathrm{EGR}}$ are, respectively, the mass of air and EGR-gas in intake manifold. $m_{\text {Exh }}$ represents the mass of exhaust gas in exhaust manifold. The other model variables and constant parameters with their value are listed in Table 4 . The temperatures $T_{\text {EGR }}$ and $T_{\text {Exh }}$ are assumed to be constant and equal to $329.436 \mathrm{~K}$ and $837 \mathrm{~K}$, respectively. $A_{\text {EGRmax }}$ is the maximum opening area for the EGR. The EGR valve (VGT) is closed when $u_{\mathrm{EGR}}=0 \%\left(u_{\mathrm{XVGT}}=0 \%\right)$ and open when $u_{\mathrm{EGR}}=$ $100 \%\left(u_{\mathrm{XVGT}}=100 \%\right)$. The static functions, $f_{\mathrm{vol}}, f_{\mathrm{EGR}}, h$, and $\tau$, are represented as interpolation in lookup tables. All these parameters are estimated using weighted least squares optimization approach. It is worth noting that the compressor and the CAC are not considered in the model as in [3] since the mass flow and the temperature after the charge-air cooler (CAC) are known variables because they are measured by the production sensors. The considered control inputs are the VGT vane position $u_{\mathrm{XVGT}}$ and the EGR valve position $u_{\mathrm{EGR}}$. Notice that the following variables: $T_{\text {CAC }}, W_{\text {HFM }}, N_{\text {Eng }}, W_{\text {Fuel }}$, and $T_{\text {Exh }}$ are considered as measurable signals. The measured outputs are the inlet and exhaust pressures.

We draw the reader's attention that another more general mean value model composed of eight states is developed, parameterized, and validated in [21]. The obtained model describes the gas flow dynamics including the dynamics in the manifold pressures, EGR, turbocharger, and actuators.

The mean value model described in the previous section was simulated and compared with real measurements obtained from the Caterpillar testbed. The results are depicted in Figures 4-7. The input variables used for the validation purpose are illustrated in Figure 4. The measured and modeled outputs $\left(P_{\text {Inlet }}\right.$ and $\left.P_{\text {Exh }}\right)$ are presented in Figure 5. The absolute value of the difference between the real and estimated variables is shown in Figure 6. One can conclude that the used model can reproduce engine dynamic behavior 


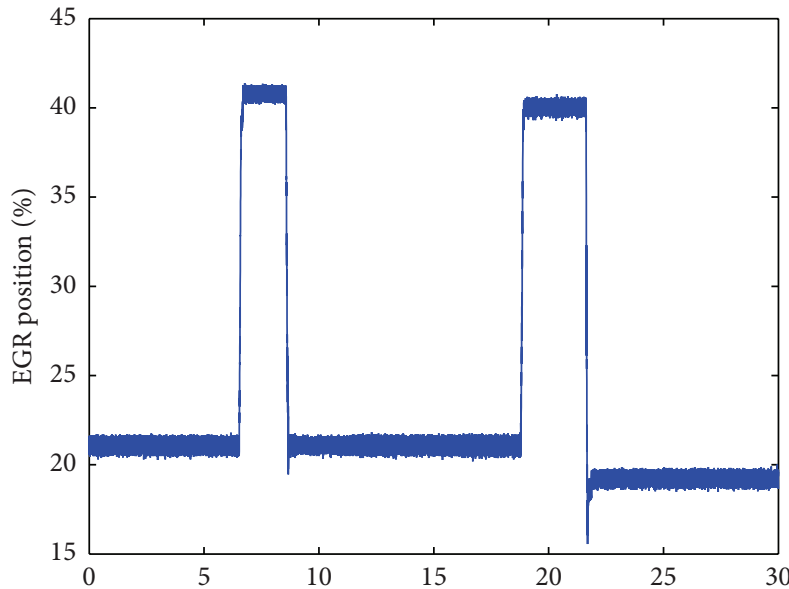

(a)

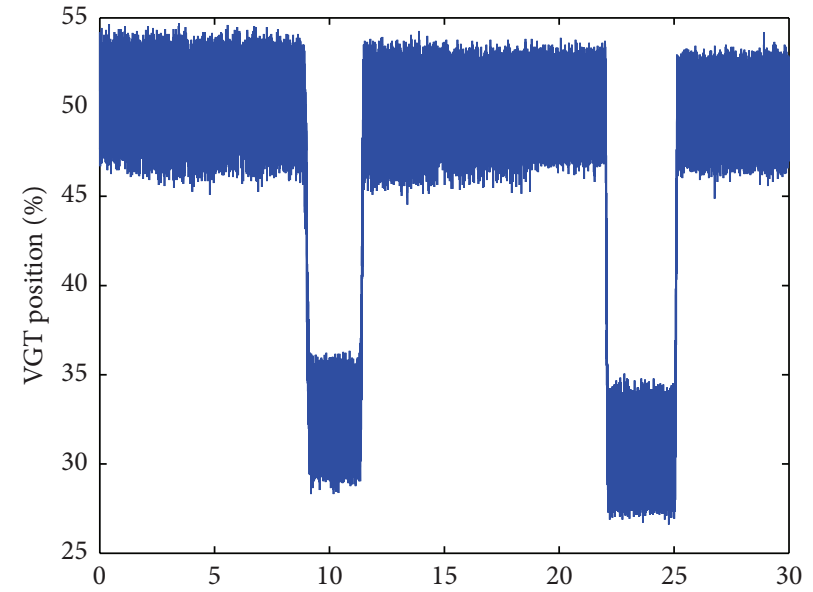

(b)

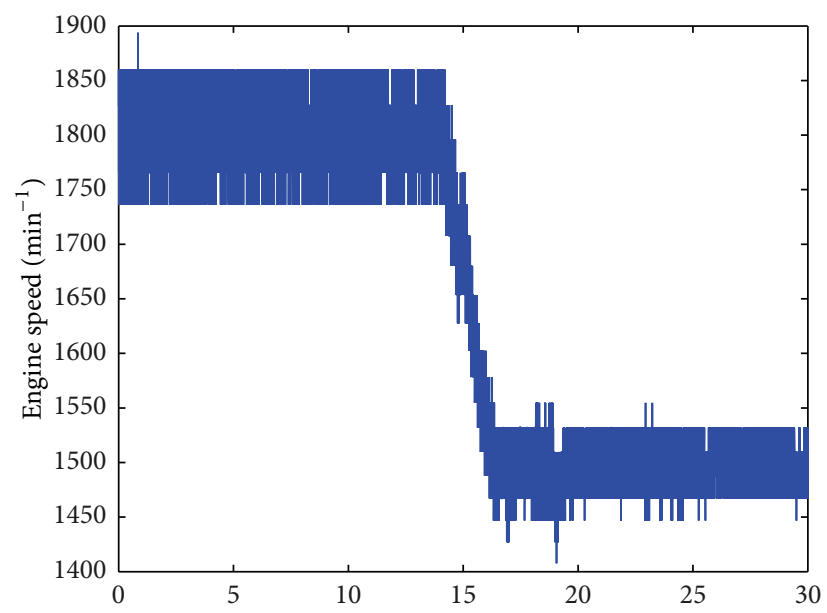

(c)

FIGURE 4: Input variables: (a) EGR position [\%], (b) VGT position [\%], and (c) engine speed [min ${ }^{-1}$ ].

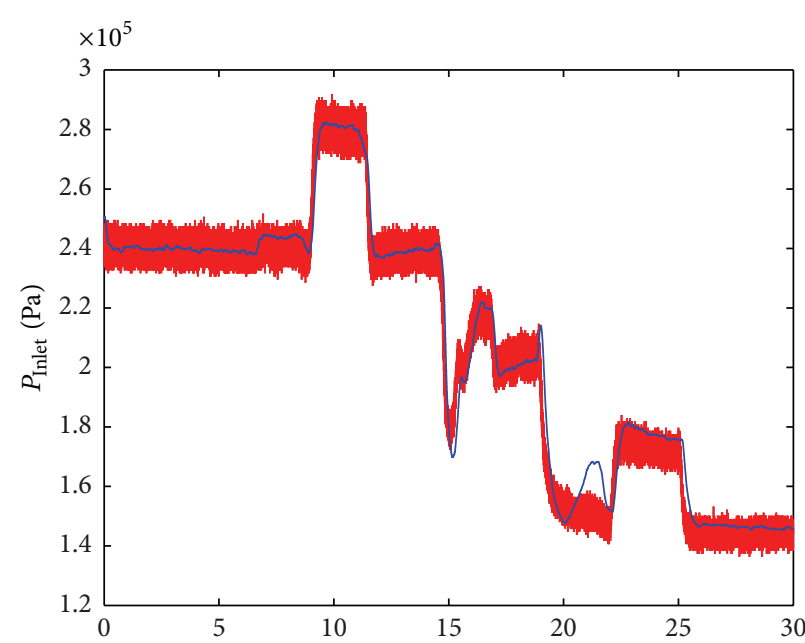

(a)

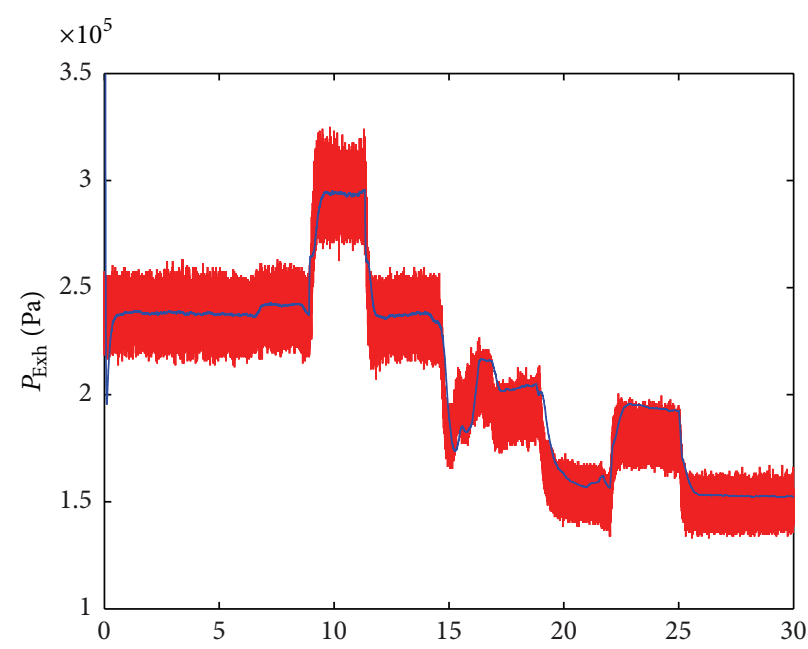

(b)

FIGURE 5: Modeled (blue color) and measured (red color) inlet and exhaust pressures. 


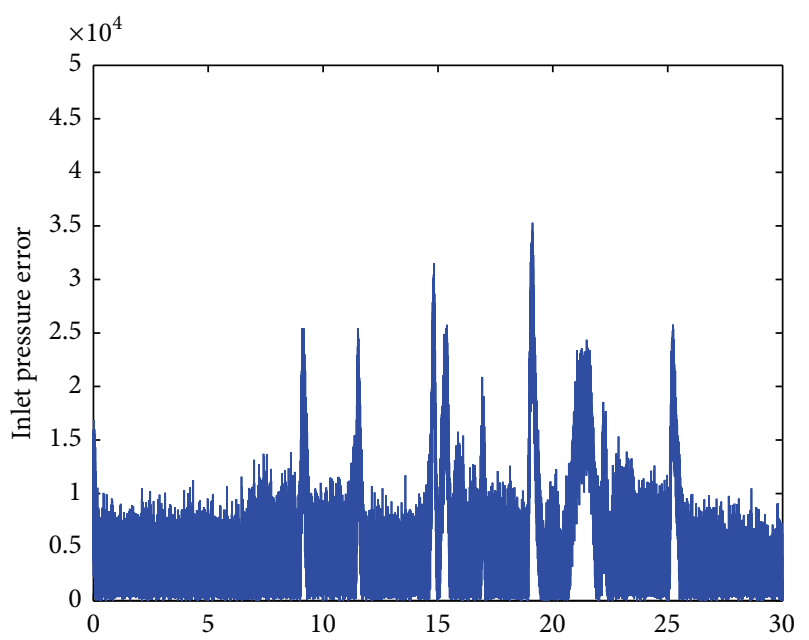

(a)

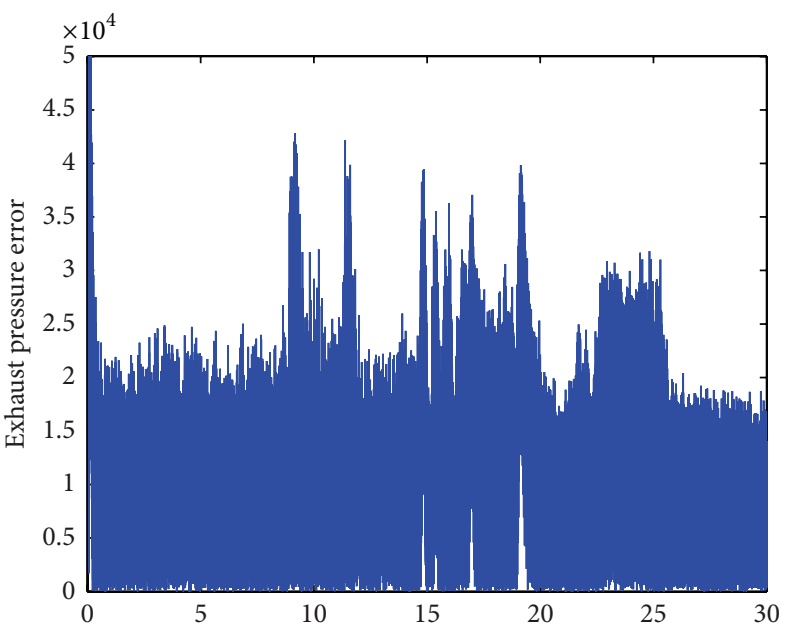

(b)

FIGURE 6: Absolute value of the difference between the measured and modeled pressures: (a) inlet pressure error and (b) exhaust pressure error.

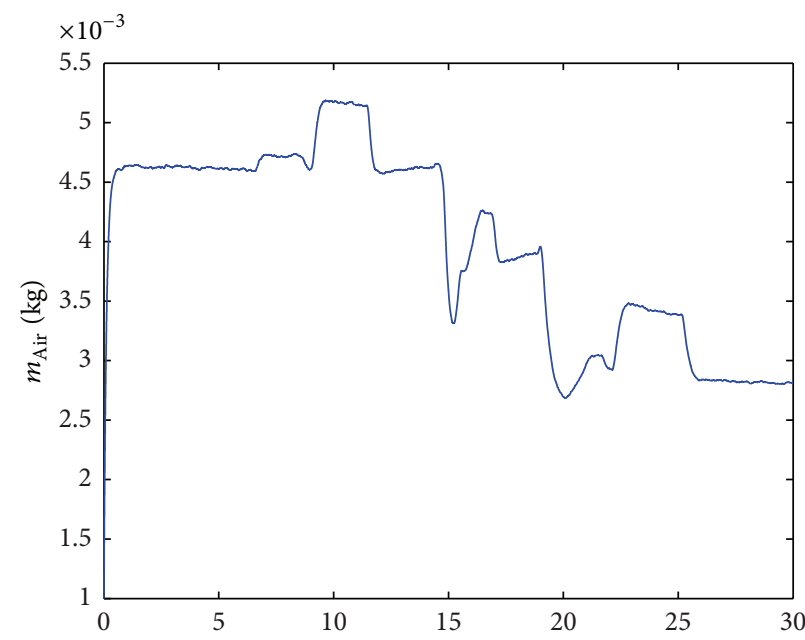

(a)

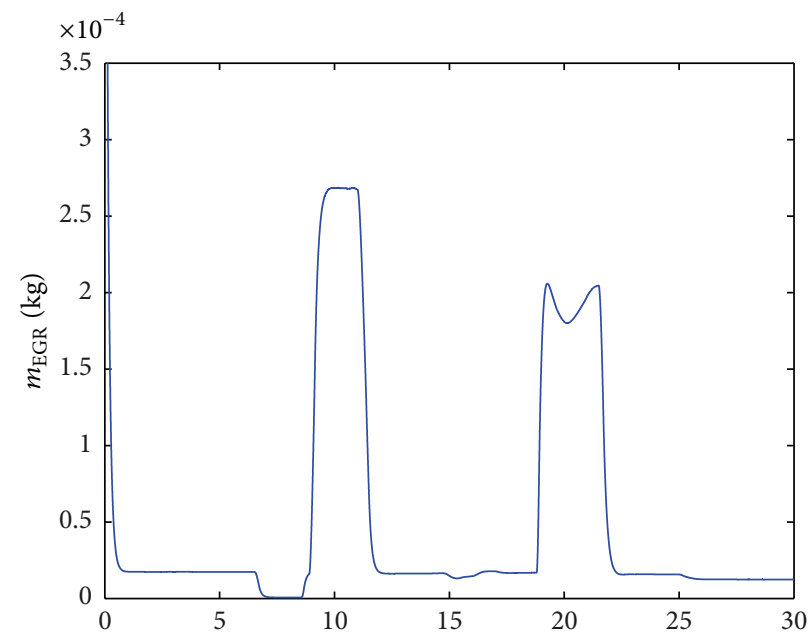

(b)

FIGURE 7: Air and EGR mass flows ( $m_{\text {Air }}$ and $\left.m_{\mathrm{EGR}}\right)$.

with good accuracy since the obtained average error is below $3 \%$. Besides, the output measurements are very noisy as shown in Figure 5. Furthermore, the air and exhaust gas mass flows $\left(m_{\text {Air }}\right.$ and $\left.m_{\text {Exh }}\right)$ are also presented in Figure 7.

\section{Nonlinear Unknown Input Observer Design}

In this section, we will present briefly a nonlinear unknown input observer developed in [12]. It was proposed for a class of Lipschitz nonlinear systems with large Lipschitz constant. The sufficient existence conditions for this observer are formulated in terms of LMIs.

Obviously, engine model (1) can be put into the form (3a) and (3b). One can see that this form contains three parts: one linear parameter varying part, a nonlinear term with known variables, and a nonlinear state-dependent part. By considering the form (3a) and (3b), this will allow us to tackle our synthesis problem based on Lyapunov theory for LPV systems. Thus, let us consider the general class of nonlinear systems described by the following equations:

$$
\begin{gathered}
\dot{x}=\sum_{j=1}^{n_{\rho}} \rho_{j} A_{j} x+B_{g} g(v, y, u)+f(x, u)+B_{f} f_{a}+B_{\bar{w}} \bar{w}, \\
y=C x+D_{\bar{w}} \bar{w}
\end{gathered}
$$

where $x \in \mathfrak{R}^{n_{x}}$ is the state vector, $u \in \mathfrak{R}^{n_{u}}$ is the control input vector, $f_{a} \in \mathfrak{R}^{n_{f a}}$ represents the actuator faults assimilated as unknown inputs, $y \in \mathfrak{R}^{n_{y}}$ is the output vector, $\bar{w} \in \mathfrak{R}^{n_{\bar{w}}}$ is the vector of disturbances/noises, and $v \in \mathfrak{R}^{n_{v}}$ is the vector of measurable signals. Notice that $v$ contains any other 
measured signals which have no link with the system output $(y)$ (e.g., temperature measurement, air mass flow $\left(W_{\mathrm{HFM}}\right)$, etc.). $A_{j}$, for $j=1, \ldots, n_{\rho}, B_{g}, B_{f}, B_{\bar{w}}, C$, and $D_{\bar{w}}$ are constant matrices with appropriate dimensions. Without loss of generality, the matrix $B_{f}$ is assumed to be of full column rank. The functions $g(v, y, u)$ and $f(x, u)$ are nonlinear. No assumption is made about the function $g(v, y, u)$. However, the function $f(x, u)$ is assumed to be once differentiable with large Lipschitz constant. The weighting functions $\rho_{j}$ are assumed to be known and depend on measurable variables. They verify the convex sum property:

$$
\sum_{j=1}^{n_{\rho}} \rho_{j}=1, \quad \rho_{j} \geq 0, \forall j \in\left\{1, \ldots, n_{\rho}\right\} .
$$

Let us first rewrite $B_{f} f_{a}$ as follows:

$$
B_{f} f_{a}=\sum_{i=1}^{n_{f_{a}}} B_{f, i} f_{a, i}
$$

where $B_{f, i}$ is the distribution matrix of the fault $f_{a, i}$.

The aim is to construct an NUIO-based residual generator which is insensitive to only one actuator fault (e.g., $B_{f, i} f_{a, i}$ ). By choosing the unknown input (scalar) $d=f_{a, i}$, the system (3a) and (3b) can be rewritten as

$$
\begin{gathered}
\dot{x}=\sum_{j=1}^{n_{\rho}} \rho_{j} A_{j} x+B_{g} g(v, y, u)+f(x, u)+B_{d} d+B_{w} w, \\
y=C x+D_{w} w
\end{gathered}
$$

where $B_{d}=B_{f, i}, B_{w}=\left[\begin{array}{ll}B_{\bar{w}} & \bar{B}_{f}\end{array}\right], D_{w}=\left[\begin{array}{ll}D_{\bar{w}} & 0\end{array}\right]$, and $w=\left[\frac{\bar{w}}{\bar{f}_{a}}\right]$ with $\bar{f}_{a}$ the actuator fault vector $f_{a}$ without the $i$ th component. $\bar{B}_{f}$ is the matrix $B_{f}$ without the $i$ th column.

It is worth noting that the necessary condition for the existence of a solution to the unknown input observer design is the following ([18, 22] for a more explanation): $\operatorname{Rank}\left(C B_{d}\right)=\operatorname{Rank}\left(B_{d}\right)$, where $B_{d}$ is a matrix of full column rank as is a column of the matrix $B_{f}$ which is assumed before to be full column rank.

The considered residual generator for the system (6a) and (6b) is given by

$$
\begin{gathered}
\dot{z}=N(\rho) z+G g(v, y, u)+M f(\widehat{x}, u)+L(\rho) y, \\
\widehat{x}=z-E y, \\
r=\Pi_{r}(y-C \widehat{x})
\end{gathered}
$$

with $N(\rho)=\sum_{j=1}^{n_{\rho}} \rho_{j} N_{j}$ and $L(\rho)=\sum_{j=1}^{n_{\rho}} \rho_{j} L_{j} . \Pi_{r}$ is a known matrix. $\widehat{x}$ represents the state estimation vector. Matrices $N$, $G, M, L$, and $E$ are the observer gains and matrices which must be determined such that $\widehat{x}$ converges asymptotically to $x$. Notice that the index $\rho$ is omitted where it is not necessary to simplify the notations.
By defining the state estimation error as $e(t)=\widehat{x}(t)-x(t)$, the error dynamics can be expressed as

$$
\begin{aligned}
\dot{e}= & N e+\left(G-M B_{g}\right) g(v, y, u)+(N M+L C-M A) x \\
& -M B_{d} d+M \widetilde{f}+\left(K D_{w}-M B_{w}\right) w-E D_{w} \dot{w}
\end{aligned}
$$

with $\tilde{f}=f(\widehat{x}, u)-f(x, u)$ and $K=L+N E$. Now, if the following matrix equations are satisfied:

$$
\begin{gathered}
N(\rho)=M A(\rho)-K(\rho) C, \quad \text { with each } N_{j} \text { stable, } \\
L(\rho)=K(\rho) \times(I+C E)-M A(\rho) E \\
G=M B_{g} \\
M=I+E C, \\
M B_{d}=0
\end{gathered}
$$

$e(t)$ goes to zero asymptotically if $w=0$ and is invariant with respect to the unknown input $d(t)$. The notation $I$ stands for the identity matrix.

Conditions $(9 \mathrm{~d})$ and $(9 \mathrm{e})$ are equivalent to $E C B_{d}=-B_{d}$. One necessary condition to have for $E C B_{d}=-B_{d}$ is that $C B_{d}$ is of full column rank since $B_{d}$ is of full column rank. If $C B_{d}$ is of full column rank, then all possible solutions of $E C B_{d}=$ $-B_{d}$ can be expressed as follows [18]:

$$
E=U+Y V
$$

with $U=-B_{d}\left(C B_{d}\right)^{\dagger}$ and $V=\left(I-C B_{d}\left(C B_{d}\right)^{\dagger}\right)$ where $Y$ can be any compatible matrix and $X^{\dagger}=\left(X^{T} X\right)^{-1} X^{T}$.

Then, the error dynamics becomes

$$
\dot{e}=(M A-K C) e+M \tilde{f}+\left(K D_{w}-M B_{w}\right) w-E D_{w} \dot{w}
$$

In order to minimize the effect of disturbances on the observer error, the $H_{\infty}$ performance criterion can be used. However, the presence of the term $\dot{w}$ makes the task difficult because it should be discarded from the derivative of the Lyapunov function as we will see later. Another solution is to add a negative term depending on $\dot{w}^{T} \dot{w}$ as proposed in [23]. This solution needs to modify the classical $H_{\infty}$ criterion. In this work, the modified $H_{\infty}$ criterion presented in [23] is used.

The modified $H_{\infty}$ estimation problem consists in computing the matrices $N$ and $L$ such that

$$
\begin{gathered}
\lim _{t \rightarrow \infty} e(t)=0 \quad \text { for } w(t)=0, \\
\|e\|_{\mathfrak{Q}_{2}^{n_{x}} \leq \gamma\|w\|_{1,2}^{r}} \text { for } w(t) \neq 0 ; e(0)=0,
\end{gathered}
$$

where $\|\cdot\|_{k, p}^{r}$ represents the Sobolev norm (see [23]).

Then to satisfy (12a)-(12b), it is sufficient to find a Lyapunov function $\Upsilon$ such that

$$
\Gamma=\dot{\Upsilon}+e^{T} e-\gamma^{2} w^{T} w-\gamma^{2} \dot{w}^{T} \dot{w}<0,
$$


where $\Upsilon=e^{T} P e$ with $P$ a positive definite symmetric matrix. From (11), $\Gamma$ is given by

$$
\begin{aligned}
\Gamma= & e^{T}\left((M A-K C)^{T} P+P(M A-K C)\right) e \\
& +e^{T} P M \tilde{f}+(M \widetilde{f})^{T} P e \\
& +e^{T} P\left(K D_{w}-M B_{w}\right) w+w^{T}\left(K D_{w}-M B_{w}\right)^{T} P e \\
& -e^{T} P E D_{w} \dot{w} \\
& -\dot{w}^{T}\left(E D_{w}\right)^{T} P e+e^{T} e-\gamma^{2} w^{T} w-\gamma^{2} \dot{w}^{T} \dot{w} .
\end{aligned}
$$

Before introducing our main result, let us present the modified mean value theorem [24] for a vector function.

Theorem 1 (see [24]). Let the canonical basis of the vectorial space $\mathfrak{R}^{s}$ for all $s \geq 1$ be defined by

$$
\begin{aligned}
& E_{s} \\
& =\left\{e_{s}(i) \mid e_{s}(i)=(\underbrace{0, \ldots, 0, \stackrel{i t h}{1}_{1}, \ldots, 0}_{s \text { components }})^{T}, i=1 \cdots s\right\} .
\end{aligned}
$$

Let $f(x): \Re^{n} \rightarrow \Re^{n}$ be a vector function continuous on $[a, b] \in \Re^{n}$ and differentiable on convex hull of the set $(a, b)$. For $s_{1}, s_{2} \in[a, b]$, there exist $\delta_{i j}^{\max }$ and $\delta_{i j}^{\min }$ for $i=1 \cdots n$ and $j=1 \cdots n$ such that

$$
\begin{array}{r}
f\left(s_{2}\right)-f\left(s_{1}\right) \\
=\left[\left(\sum_{i, j=1}^{n, n} H_{i j}^{\max } \delta_{i j}^{\max }\right)+\left(\sum_{i, j=1}^{n, n} H_{i j}^{\min } \delta_{i j}^{\min }\right)\right]\left(s_{2}-s_{1}\right) \\
\delta_{i j}^{\max }, \delta_{i j}^{\min } \geq 0, \quad \delta_{i j}^{\max }+\delta_{i j}^{\min }=1,
\end{array}
$$

where

(i) $h_{i j}^{\max } \geq \max \left(\partial f_{i} / \partial x_{j}\right)$ and $h_{i j}^{\min } \leq \min \left(\partial f_{i} / \partial x_{j}\right)$,

(ii) $H_{i j}^{\max }=e_{n}(i) e_{n}^{T}(j) h_{i j}^{\max }$ and $H_{i j}^{\min }=e_{n}(i) e_{n}^{T}(j) h_{i j}^{\min }$.

The proof of this theorem is given in [24].

In our case, the nonlinear function $f$ depends on the state vector $x$ and also on the known input $u$. Although the previous theorem is applicable in our case, $u$ is bounded.

Now, we can give a sufficient condition under which the observer given by (7a), (7b), and (7c) is a NUIO. Thus, the negativity of $\Gamma$ is ensured by the following theorem.

Theorem 2 (see [25]). The observer error $e(t)$ converges asymptotically towards zero if there exist matrices $\bar{K}_{k}, \bar{Y}$, a positive definite symmetric matrix $P$, and a positive scalar $\mu$ such that the following LMIs are satisfied:

$$
P>0 \text {, }
$$

$$
\begin{aligned}
& {\left[\begin{array}{ccc}
\Xi_{i j k}^{\max } & \Phi_{k} & -P Y D_{w}-\bar{Y} V D_{w} \\
(*) & -\mu I & 0 \\
(*) & (*) & -\mu I
\end{array}\right]<0,} \\
& {\left[\begin{array}{ccc}
\Xi_{i j k}^{\min } & \Phi_{k} & -P Y D_{w}-\bar{Y} V D_{w} \\
(*) & -\mu I & 0 \\
(*) & (*) & -\mu I
\end{array}\right]<0}
\end{aligned}
$$

$\forall i=1, \ldots, n, j=1, \ldots, n$ and $k=1, \ldots, n_{\rho}$, where

$$
\begin{aligned}
\Phi_{k}=-\bar{K}_{k} D_{w} & +P(I+U C) B_{w}+\bar{Y} V C B_{w}, \quad \mu=\gamma^{2}, \\
\Xi_{i j k}^{\max }= & {\left[(I+U C)\left(A+\bar{H}_{i j}^{\max }\right)\right]^{T} P } \\
& +P(I+U C)\left(A+\bar{H}_{i j}^{\max }\right)-C^{T} \bar{K}_{k}^{T} \\
& -\bar{K}_{k} C+\left(A+\bar{H}_{i j}^{\max }\right)^{T} C^{T} V^{T} \bar{Y}^{T} \\
& +\bar{Y} V C\left(A+\bar{H}_{i j}^{\max }\right)+I, \\
\Xi_{i j k}^{\min }= & {\left[(I+U C)\left(A+\bar{H}_{i j}^{\min }\right)\right]^{T} P } \\
& +P(I+U C)\left(A+\bar{H}_{i j}^{\min }\right)-C^{T} \bar{K}_{k}^{T} \\
& -\bar{K}_{k} C+\left(A+\bar{H}_{i j}^{\min }\right)^{T} C^{T} V^{T} \bar{Y}^{T} \\
& +\bar{Y} V C\left(A+\bar{H}_{i j}^{\min }\right)+I, \\
\bar{H}_{i j}^{\max }= & Z_{H} H_{i j}^{\max }, \quad \bar{H}_{i j}^{\min }=Z_{H} H_{i j}^{\min }
\end{aligned}
$$

with $Z_{H}=n \times n$. Solving LMIs (17a)-(17c) leads to determine matrices $P, \bar{Y}$, and $\bar{K}_{k}$. The matrices $K_{k}$ and $Y$ can be obtained from $K_{k}=P^{-1} \bar{K}_{k}$ and $Y=P^{-1} \bar{Y}$. The other matrices $N$ and $L$ can then be deduced easily from (9a) and (9b), respectively.

Proof. The proof is omitted. A sketch of this proof is presented in [25].

Notice that if there exist terms such that $\partial f_{i} / \partial x_{j}=0$, then the scaling factor $\sum_{i, j=1}^{n, n}\left(\delta_{i j}^{\max }+\delta_{i j}^{\min }\right)$ is less than 1 . Consequently, the scaling factor $\bar{Z}_{H}$ must be redefined as follows:

$$
\begin{gathered}
\bar{Z}_{H}=\sum_{i, j=1}^{n, n}\left(\delta_{i j}^{\max }+\delta_{i j}^{\min }\right)=n \times n-n_{0}, \\
\frac{\sum_{i, j=1}^{n, n}\left(\delta_{i j}^{\max }+\delta_{i j}^{\min }\right)}{\bar{Z}_{H}}=1,
\end{gathered}
$$

where $n_{0}$ is the number of terms in $\partial f_{i} / \partial x_{j}$ that equal zero. 


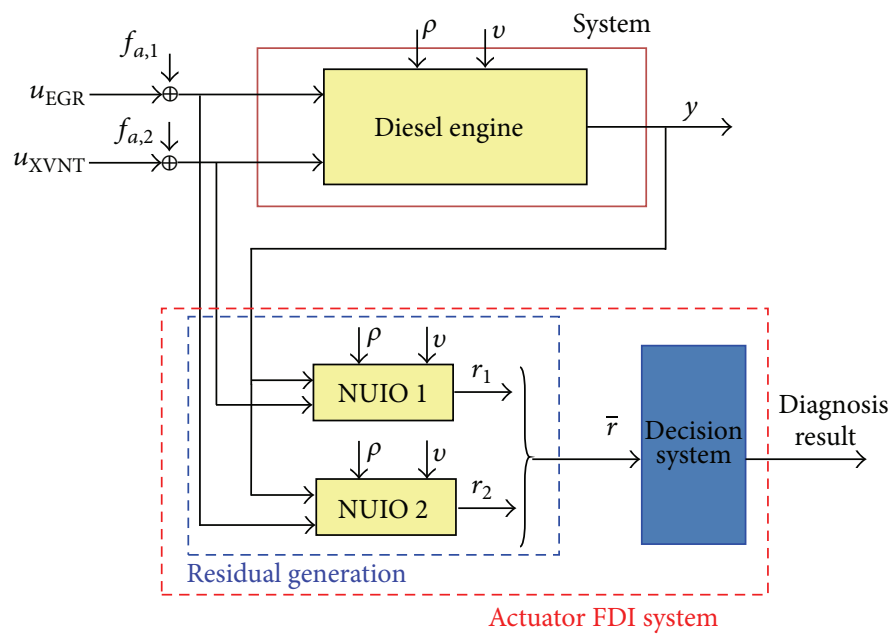

FIGURE 8: FDI system.

The procedure to design the NUIO parameters is summarized by the following algorithm.

Algorithm 3. NUIO design is as follows:

(1) compute $U$ and $V$ from (10),

(2) determine matrices $Y$ and $K_{k}$, with $k=1, \ldots, n_{\rho}$ from the LMI sets $(17 \mathrm{a})-(17 \mathrm{c})$,

(3) compute the observer matrices $E, M, G, N$, and $L$ from (10), (9d), (9c), (9b), and (9a), respectively.

\section{Residual Generation}

This section addresses the problem of actuator faults isolation based on a bank of residual generators (see Figure 8). Each residual on the proposed scheme is an NUIO based on model (6a) and (6b). Notice that the system (1) can be easily rewritten in a state space form as (3a) and (3b) (see Appendix C). Furthermore, each residual is designed to be insensitive to only one fault. Thus, the actuator faults can be easily isolated since only one residual goes to zero while the others do not. Therefore, for our application, the combination of two observers is sufficient to detect and isolate any faulty actuator. It is also assumed that only a single actuator fault can occur at one time.

To construct the bank of residual generators we have to

(i) design an NUIO which generates the residual $r_{1}$ insensitive to $f_{a, 1}$ by taking

$B_{d}=\left[\begin{array}{llll}\frac{R_{\mathrm{Exh}} T_{\mathrm{EGR}} A_{\mathrm{EGRmax}} c_{p, \mathrm{Exh}}}{V_{\text {Inlet }} c_{v, \text { Exh }}} & 0 & A_{\mathrm{EGRmax}} & -A_{\mathrm{EGRmax}}\end{array}\right]^{T}$,

(ii) design an NUIO which generates the residual $r_{2}$ insensitive to $f_{a, 2}$ by taking

$$
B_{d}=\left[\begin{array}{llll}
0 & 0 & 0 & 1
\end{array}\right]^{T} \text {. }
$$

TABLE 2: Effect of the faults on the residuals.

\begin{tabular}{lcc}
\hline $\bar{r}$ & $f_{1}$ & $f_{2}$ \\
\hline$r_{1}$ & 0 & $\times$ \\
$r_{2}$ & $\times$ & 0 \\
\hline
\end{tabular}

To obtain $B_{d}$ as in (20), it suffices to replace in the original system the input vector $u$ by $u+f_{a}$, where $f_{a}$ represents the actuator fault vector. Thus, $B_{d}$ can be obtained easily by gathering the terms multiplying the vector $f_{a}$ in one matrix.

Each residual, $r_{i}(t)(i=1,2)$, computed from (7a), (7b), and $(7 c)$, is a 1-dimensional vector associated with one of the actuators $u_{\mathrm{EGR}}$ and $u_{\mathrm{XVGT}}$. By stacking the two vectors, one defines $\bar{r}(t)=\left[r_{1}^{\prime}(t), r_{2}^{\prime}(t)\right]^{\prime}$. In the absence of faults, this vector of residuals has zero mean, while, upon occurrence of a single step-like fault, the effect on each of its components is indicated in Table 2. A " $x$ " is placed when the fault located in the corresponding column affects the mean of the residual component on the corresponding row, and a " 0 " when the residual component presents very low sensitivity to the fault. The sampled vector $\bar{r}(k)$ can be rewritten as

$$
\bar{r}(k)=\bar{r}_{0}(k)+\sum_{\ell=1}^{2} v_{\ell} \Gamma_{\ell}(k) 1_{\left\{k \geq k_{0}\right\}} \delta_{\ell},
$$

where the sample number $k$ corresponds to the time instant $t=k T_{s}$ with $T_{s}$ denoting the sampling period, $\bar{r}_{0}(k)$ is the fault-free residual, $\Gamma_{\ell}(k)$ is the dynamic profile of the change on $\bar{r}(k)$ due to a unit step-like fault $f_{\ell}$, and $\nu_{\ell}$ is the magnitude of fault $\ell$. Model (22) will be used as the basis for the decision system design in the next section.

One can see that residuals are designed in a deterministic framework. However, due to measurement noise and system disturbances, parameters uncertainties, and variations, the computed residuals are stochastic signals. As a consequence, a multi-CUSUM method will be used for the decision step. Another way would have been to consider the noise and stochastic disturbances characteristics to design robust residuals, analyze the stochastic characteristics of the residuals, 
TABLE 3: Caterpillar 3126B engine characteristics.

Description

\begin{tabular}{lc} 
Model & Caterpillar 3126B \\
Type of engine & Inline, 4-stroke \\
Number of cylinders & 6 \\
Number of inlet valves & 2 \\
Number of exhaust valves & 1 \\
Firing order & $1-5-3-6-2-4$ \\
Type of combustion & Direct injection \\
Maximum torque & $1166 \mathrm{Nm} @ 1440 \mathrm{rpm}$ \\
Maximum power & $224 \mathrm{kw} @ 2200 \mathrm{rpm}$ \\
Idle speed & $700 \mathrm{rpm}$ \\
Maximum speed & $2640 \mathrm{rpm}$ \\
\hline
\end{tabular}

Maximum speed

Geometrical characteristics

Bore

$110 \mathrm{~mm}$

Stroke

Compression ratio

$127 \mathrm{~mm}$

Total displacement

16

Connecting rod length

7.25 liter

Crank throw radius

$199.9 \mathrm{~mm}$

$63.5 \mathrm{~mm}$

Injection system

Type

HEUI

Injection pressure

Injection orifices number

200-145 bar

Type of combustion

6

Direct injection

Geometrical characteristics of manifolds and pipes

Intake manifold

$5 \mathrm{~L}$

Exhaust manifold

$0.945 \mathrm{~L}$

and design the decision algorithm with respect to these characteristics. However, this would be too complex to do for our application and would lie anyway on given assumptions on the stochastic disturbances.

\section{Decision System}

In this section, we will present a decision system based on a statistical approach proposed in [26]. This approach, named multi-CUSUM, was developed in [27] and refined in [28]. Indeed, in model (22), it is seen that an actuator fault induces a change in the mean of the residual vector $\bar{r}(k)$. Thus, the problem amounts to detecting and isolating a step-like signal within a white Gaussian noise sequence. Therefore, the change detection/isolation problem can be stated as the following hypothesis testing:

$$
\begin{aligned}
\mathscr{H}_{0}: \mathscr{L}(\bar{r}(i))=\mathscr{N}\left(\boldsymbol{\mu}_{0}, \Sigma\right), & i=1, \ldots, k, \\
\mathscr{H}_{\ell}: \mathscr{L}(\bar{r}(i))=\mathscr{N}\left(\boldsymbol{\mu}_{0}, \Sigma\right), & i=1, \ldots, k_{0}-1, \\
\mathscr{L}(\bar{r}(i))=\mathscr{N}\left(\boldsymbol{\mu}_{\ell}, \Sigma\right), & i=k_{0}, \ldots, k,
\end{aligned}
$$

where $k_{0} \in[1, k]$, and $\boldsymbol{\mu}_{\ell}=\boldsymbol{\mu}_{0}+\nu_{\ell} \Gamma_{\ell}$ is the mean value of the residual sequence when the $\ell$ th fault has occurred, for $\ell \in\{1,2\}$. The decision system is based on a combination of CUSUM decision functions [27], each of them involving the log-likelihood ratio between hypotheses $\mathscr{H}_{\ell}$ and $\mathscr{H}_{0}$, namely,

$$
s_{k}(\ell, 0)=\ln \frac{p_{\ell}(\bar{r}(k))}{p_{0}(\bar{r}(k))},
$$

where $p_{\ell}$ is the probability density function of $\bar{r}(k)$ under the hypothesis $\mathscr{H}_{\ell}$. Under the Gaussian hypothesis, the loglikelihood ratio can be rewritten as

$$
s_{k}(\ell, 0)=\left(\boldsymbol{\mu}_{\ell}-\boldsymbol{\mu}_{0}\right)^{T} \Sigma^{-1}\left(\bar{r}(k)-\frac{1}{2}\left(\boldsymbol{\mu}_{\ell}+\boldsymbol{\mu}_{0}\right)\right) .
$$

The CUSUM decision function is defined recursively as

$$
g_{k}(\ell, 0)=\max \left(0, g_{k-1}(\ell, 0)+s_{k}(\ell, 0)\right)
$$

In order to estimate the fault occurrence time, the number of successive observations for which the decision function remains strictly positive is computed as $N_{\ell}(k)=N_{\ell}(k-$ $1) 1_{\left\{g_{k-1}(\ell, 0)>0\right\}}+1$. To decide whether fault $\ell$ has occurred, one has to check that, on average, all the likelihood ratios $s_{k}(\ell, j)$, for $1 \leq j \neq \ell \leq 2$, are significantly larger than zero. Noticing that $s_{k}(\ell, j)=s_{k}(\ell, 0)-s_{k}(j, 0)$, one can build a CUSUM algorithm to decide between hypotheses $\mathscr{H}_{\ell}$ and $\mathscr{H}_{j}$ by taking into account the difference between $g_{k}(\ell, 0)$ and $g_{k}(j, 0)$. Hence a decision that $\mathscr{H}_{\ell}$ holds can be issued when the following decision function becomes positive:

$$
\bar{g}_{\ell}=\min _{0 \leq j \neq \ell \leq 2}\left(g_{k}(\ell, 0)-g_{k}(j, 0)-h_{\ell, j}\right)
$$

for $\ell=1,2$ and $g_{k}(0,0)=0$.

Like in $[26,27]$, it is advisable to consider only two values for the thresholds $h_{\ell, j}$, for each $\ell=1, \ldots, n_{f}$, namely,

$$
h_{\ell, j}= \begin{cases}h_{\ell d}, & \text { for } j=0, \\ h_{\ell i}, & \text { for } 1 \leq j \neq \ell \leq n_{f},\end{cases}
$$

where $h_{\ell d}$ is the detection threshold and $h_{\ell i}$ is the isolation threshold. Notice that the mean detection delay, the mean time before false alarm, and the probability of a false isolation depend on the choice of these thresholds. Indeed, the thresholds $h_{\ell d}$ and $h_{\ell i}$ can be linked to the mean detection delay $(\bar{\tau})$ for the fault $\ell$ thanks to the following expression [27] assuming that $h_{\ell d}=\bar{\gamma} h_{\ell i}$ where $\bar{\gamma} \geq 1$ is a constant:

$$
\bar{\tau}=\max \left(\frac{h_{\ell d}}{\bar{\kappa}_{\ell, 0}}, \frac{h_{\ell i}}{\min _{j \neq 0, \ell}\left(\bar{\kappa}_{\ell, j}\right)}\right) \quad \text { as } h_{\ell i} \longrightarrow \infty \text {, }
$$

where $\bar{\kappa}_{\ell, j}$ is the Kullback-Leibler information defined as

$$
\bar{\kappa}_{\ell, j}=\frac{1}{2}\left(\boldsymbol{\mu}_{\ell}-\boldsymbol{\mu}_{j}\right)^{T} \Sigma^{-1}\left(\boldsymbol{\mu}_{\ell}-\boldsymbol{\mu}_{j}\right)
$$


TABLE 4: The variables used in the engine model.

\begin{tabular}{|c|c|c|}
\hline Symb. & Quantity & Value/unit \\
\hline$P_{\text {Inlet }}$ & Pressure in intake manifold & $\mathrm{Pa}$ \\
\hline$p_{\text {Atm }}$ & Atmospheric pressure & $\mathrm{Pa}$ \\
\hline$W_{\text {Turb }}$ & Exhaust mass flow past the turbine & $\mathrm{kg} \cdot \mathrm{s}^{-1}$ \\
\hline$u_{\mathrm{XVNT}}$ & Position of VNT vanes & $\%$ \\
\hline$N_{\text {Eng }}$ & Engine speed & $\min ^{-1}$ \\
\hline$W_{\text {Fuel }}^{\circ}$ & Mass flow of injected fuel & $\mathrm{kg} \cdot \mathrm{s}^{-1}$ \\
\hline$Q_{\mathrm{LHV}}$ & Lower heating value & $\mathrm{J} \cdot \mathrm{kg}^{-1}$ \\
\hline$A_{\mathrm{EGR}}$ & Effective area of EGR valve & $\mathrm{m}^{2}$ \\
\hline$R_{\text {Inlet }}$ & Gas constant in intake manifold & $\mathrm{J} \cdot(\mathrm{kg} \cdot \mathrm{K})$ \\
\hline$W_{\text {Inlet }}$ & Mass flow into engine inlet ports & $\mathrm{kg} \cdot \mathrm{s}^{-1}$ \\
\hline$T_{\text {Inlet }}$ & Temperature in the intake manifold & $\mathrm{K}$ \\
\hline$m_{\text {Air }}$ & Mass of air in intake manifold & $\mathrm{kg}$ \\
\hline$m_{\mathrm{EGR}}$ & Mass of EGR gas in intake manifold & $\mathrm{kg}$ \\
\hline$W_{\text {Exh }}$ & Exhaust mass flow into the exhaust manifold & $\mathrm{kg} \cdot \mathrm{s}^{-1}$ \\
\hline$m_{\text {Exh }}$ & Mass of exhaust gas in exhaust manifold & $\mathrm{kg}$ \\
\hline$P_{\text {Exh }}$ & Pressure in exhaust manifold & $\mathrm{Pa}$ \\
\hline$c_{p, \text { Inlet }}$ & Specific heat at const. pres. in intake manifold & $\mathrm{J} \cdot(\mathrm{kg} \cdot \mathrm{K})$ \\
\hline$c_{v, \text { Inlet }}$ & Specific heat at const. vol. in intake manifold & $\mathrm{J} \cdot(\mathrm{kg} \cdot \mathrm{K})$ \\
\hline$\kappa$ & Ratio of specific heats & $c_{p} / c_{v}$ \\
\hline$W_{\text {HFM }}$ & Air mass flow past the air mass flow sensor & $\mathrm{kg} \cdot \mathrm{s}^{-1}$ \\
\hline$W_{\mathrm{EGR}}$ & EGR mass flow into intake manifold & $\mathrm{kg} \cdot \mathrm{s}^{-1}$ \\
\hline$V_{\text {Inlet }}$ & Volume of intake manifold & $1.8 \times 10^{-3} \mathrm{~m}^{3}$ \\
\hline$R_{\text {Air }}$ & Gas constant of air & $288.2979 \mathrm{~J} \cdot(\mathrm{kg} \cdot \mathrm{K})$ \\
\hline$c_{p, \text { Air }}$ & Specific heat at const. pres. of air & $1067.4 \mathrm{~J} \cdot(\mathrm{kg} \cdot \mathrm{K})$ \\
\hline$c_{v, \text { Air }}$ & Specific heat at const. vol. of air & $779.1021 \mathrm{~J} \cdot(\mathrm{kg} \cdot \mathrm{K})$ \\
\hline$R_{\text {Exh }}$ & Gas constant of exhaust gas of exhaust gas & $290.155 \mathrm{~J} \cdot(\mathrm{kg} \cdot \mathrm{K})$ \\
\hline$c_{p, \mathrm{Exh}}$ & Specific heat at const. pres. of exhaust gas & $1288.08 \mathrm{~J} \cdot(\mathrm{kg} \cdot \mathrm{K})$ \\
\hline$c_{v, \text { Exh }}$ & Specific heat at const. vol. of exhaust gas & $997.9250 \mathrm{~J} \cdot(\mathrm{kg} \cdot \mathrm{K})$ \\
\hline$T_{\mathrm{CAC}}$ & Temperature of the air after the charge-air cooler & $322.5 \mathrm{~K}$ \\
\hline$T_{\mathrm{EGR}}$ & Temperature of EGR gas flow into the i.m. & $322 \mathrm{~K}$ \\
\hline$T_{\text {Exh }}$ & Temperature in exhaust manifold & $837 \mathrm{~K}$ \\
\hline$V_{\text {Eng }}$ & Engine displacement & $7.239 \times 10^{-3} \mathrm{~m}^{3}$ \\
\hline$V_{\text {Exh }}$ & Volume of exhaust manifold & $9.4552 \times 10^{-3} \mathrm{~m}^{3}$ \\
\hline
\end{tabular}
(27):

When considering $h_{\ell, d}=h_{\ell, i}=h_{\ell}$, (31) is used instead of

$$
g_{\ell}^{*}=\min _{0 \leq j \neq \ell \leq 2}\left(g_{k}(\ell, 0)-g_{k}(j, 0)\right)
$$

and an alarm is generated when a $g_{\ell}^{*} \geq h_{\ell}$.

The reader can refer to $[26,27]$ for more detailed information.

\section{Experimental Results and Discussion}

The proposed fault detection and isolation approach has been tested on the caterpillar 3126 engine located at Sussex University, UK. The aim is to detect and isolate any additive actuator fault. The detection delay $\bar{\tau}$ should be lower than $0.01 \mathrm{~s}$ in average. This delay is defined as the difference between the alarm time and the actual fault occurrence time.
7.1. Design Parameters. For the design of the residual generators, the gain matrices given by (9a)-(9e) should be determined. Unfortunately, we cannot present their values due to pages limitation. Notice that the LMIs (17a)-(17c) are solved by using the software YALMIP toolbox, which is a toolbox for modeling and optimization in Matlab. Matrix $\Pi_{r}$ for the two residual generators is chosen as $\Pi_{r}=[0,1]$. The sampling period $T_{s}$ is set as $10^{-3} \mathrm{~s}$.

Remark 4. It is worth noting that no feasible solution is obtained with the approach presented in [18] due to the Lipschitz constant value of the nonlinear function $f$ (see (C.7)). In fact, the Lipschitz constant value is bigger than $10^{10}$ in our case. Other methods can be used, or extended to our case, as that proposed in [23] for estimating the state and unknown input vectors. Unfortunately, these methods are computationally demanding since the number of LMIs, 

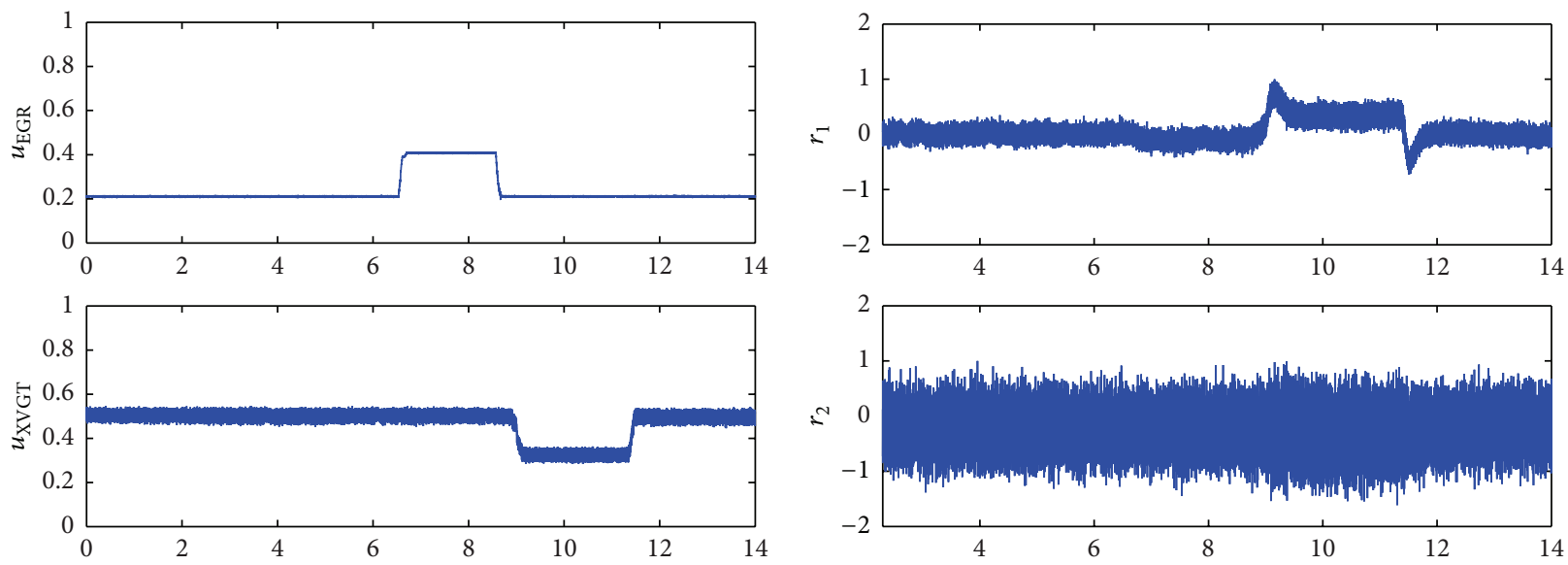

(a)

(b)
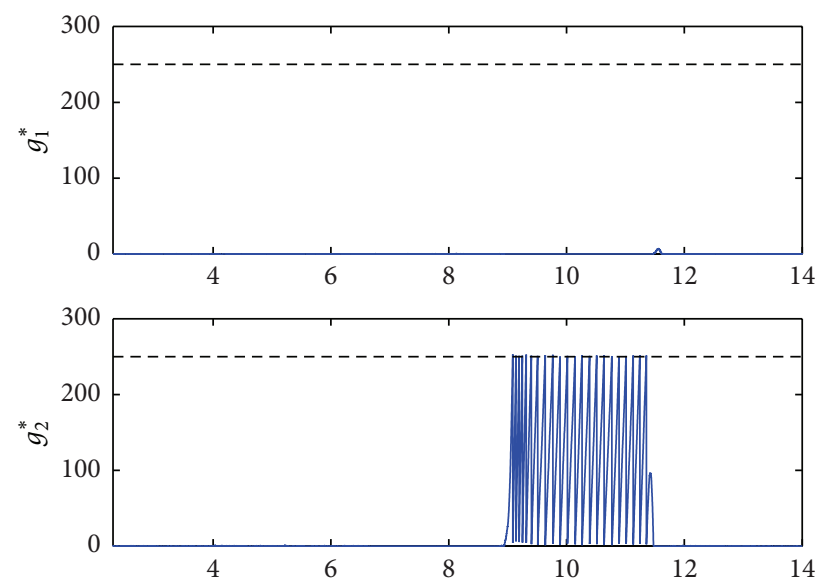

(c)

FIGURE 9: Experimental results: (a) EGR and VGT actuators, (b) residuals $\left(r_{i}\right)$, and (c) multi-CUSUM decision functions.

in each residual generator, that should be solved is equal to $N_{\text {LMI }}=2^{n \times n_{f}}$, where $n_{f}$ is the number of nonlinearities in the system. So, in our case $N_{\text {LMI }}=2^{4 \times 4}=65536$. In addition, it is only proposed for the standard systems with a linear timeinvariant part $(A x)$. We know that the number of LMIs will be strongly increased if this method is extended to our LPV case.

The covariance matrix $\Sigma$ and the mean $\boldsymbol{\mu}_{0}$ used for designing the multi-CUSUM algorithm are estimates of the variance and the mean of $\bar{r}$ obtained from a set of simulation data generated in healthy operating conditions. Assuming that all considered faults should be detected and isolated with a mean detection delay $\bar{\tau} \leq 0.1 \mathrm{~s}$, the threshold is selected as $h_{\ell}=1000$.

7.2. Validation Step. For illustrating the performance of this approach, the following scenario is considered. First, a positive step-like change in the EGR actuator appears when $t \in[6.5,8] \mathrm{s}$. Next, a single negative step-like fault in the VGT actuator is introduced in the time interval $[8.9,11.5] \mathrm{s}$.

The experiment is performed with engine average speed $N_{\text {Eng }}=1800 \mathrm{rpm}$, where the minimum and maximum value of $N_{\text {Eng }}\left(\underline{N}_{\text {Eng }}\right.$ and $\left.\bar{N}_{\text {Eng }}\right)$ are $1700 \mathrm{rpm}$ and $1900 \mathrm{rpm}$, respectively. The initial conditions for the two observers are randomly chosen as follows:

$$
\begin{aligned}
& z 1_{0}=\left[\begin{array}{llll}
12 & 0 & 2 \times 10^{-7} & 10^{-10}
\end{array}\right]^{T}, \\
& z 2_{0}=\left[\begin{array}{llll}
12 & 0 & 2 \times 10^{-7} & 10^{-10}
\end{array}\right]^{T} .
\end{aligned}
$$

The experimental results are shown in Figure 9. First, the actuators $\left(u_{\mathrm{EGR}}\right.$ and $\left.u_{\mathrm{XVGT}}\right)$ behavior is illustrated in Figure 9(a). Then, the normalized residuals $r_{1}$ and $r_{2}$ are shown in Figure 9(b). Finally, the decision functions resulting from the multi-CUSUM algorithm are presented in Figure 9(c). It is clear from the last figures (Figure 9(c)) that only the exact decision function $g_{2}^{*}$, corresponding to an occurred fault in $u_{\mathrm{XVGT}}$, crosses the threshold. It means that this fault is correctly detected and isolated. The obtained mean detection delay $\bar{\tau}$, given by (29), is equal to $0.0890 \mathrm{~s}$. As expected, this fault is detected and isolated with a mean detection delay $\bar{\tau} \leq 0.1 \mathrm{~s}$. 


\section{Conclusion}

The problem of actuator fault detection and isolation for diesel engines is treated in this paper. The faults affecting the EGR system and VGT actuator valves are considered. A bank of NUIO has been used. Each residual is designed to be insensitive to only one fault. By using this kind of scheme and by assuming that only a single fault can occur at one time, each actuator fault can be easily isolated since only one residual goes to zero while the others do not. A multi-CUSUM algorithm for statistical change detection and isolation is used as a decision system. Fault detection/isolation is achieved within the imposed timeslot. Experimental results are presented to demonstrate the effectiveness of the proposed approach.

\section{Appendices}

\section{A. Engine Characteristics}

See Table 3.

\section{B. Nomenclature}

See Table 4.

\section{Diesel Engine Model}

The system (1) can be easily rewritten in state space form as (3a) and (3b). Indeed, it suffices to extract the linear part by doing product development and to separate the nonlinear part with known or measurable variables $(v, y, u)$. The rest is gathered in the general nonlinear part $(f(x, u))$. The state, known input and output vectors, and the variables $\rho$ and $v$ are defined as

$$
\begin{aligned}
& x=\left[\begin{array}{llll}
P_{\text {Inlet }} & m_{\text {Air }} & m_{\mathrm{EGR}} & m_{\mathrm{Exh}}
\end{array}\right]^{T}, \\
& u=\left[\begin{array}{ll}
u_{\mathrm{EGR}} & u_{\mathrm{XVNT}}
\end{array}\right]^{T}, \\
& y=\left[\begin{array}{ll}
P_{\text {Inlet }} & P_{\text {Exh }}
\end{array}\right]^{T}, \\
& \rho=N_{\text {Eng }}, \\
& v=\left[\begin{array}{lll}
T_{\mathrm{CAC}} & W_{\mathrm{HFM}} & W_{\text {Fuel }}
\end{array}\right]^{T} .
\end{aligned}
$$

The matrices $A_{1}$ and $A_{2}$ are given by

$A_{1}$

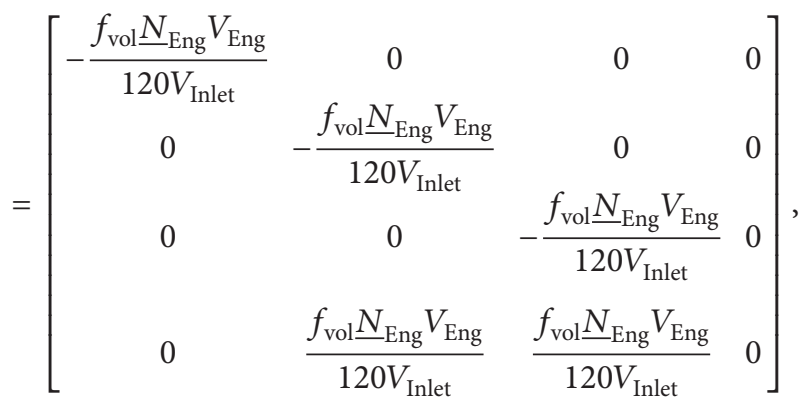

$$
\begin{aligned}
& A_{2} \\
& =\left[\begin{array}{cccc}
-\frac{f_{\text {vol }} \bar{N}_{\text {Eng }} V_{\text {Eng }}}{120 V_{\text {Inlet }}} & 0 & 0 & 0 \\
0 & -\frac{f_{\text {vol }} \bar{N}_{\text {Eng }} V_{\text {Eng }}}{120 V_{\text {Inlet }}} & 0 & 0 \\
0 & 0 & -\frac{f_{\text {vol }} \bar{N}_{\text {Eng }} V_{\text {Eng }}}{120 V_{\text {Inlet }}} & 0 \\
0 & \frac{f_{\text {vol }} \bar{N}_{\text {Eng }} V_{\text {Eng }}}{120 V_{\text {Inlet }}} & \frac{f_{\text {vol }} \bar{N}_{\text {Eng }} V_{\text {Eng }}}{120 V_{\text {Inlet }}} & 0
\end{array}\right] \text {, }
\end{aligned}
$$

where $\underline{N}_{\text {Eng }}$ and $\bar{N}_{\text {Eng }}$ are, respectively, the minimum and maximum value of the measurable variable $N_{\text {Eng }} \cdot \rho_{1}$ and $\rho_{2}$ are defined as

$$
\rho_{1}=\frac{\bar{N}_{\text {Eng }}-N_{\text {Eng }}}{\bar{N}_{\text {Eng }}-\underline{N}_{\text {Eng }}}, \quad \rho_{2}=\frac{N_{\text {Eng }}-\underline{N}_{\text {Eng }}}{\bar{N}_{\text {Eng }}-\underline{N}_{\text {Eng }}} .
$$

The matrices $B_{g}, C$, and $B_{\bar{w}}$ are expressed as

$$
\begin{gathered}
B_{g}=\left[\begin{array}{ccc}
\frac{R_{\text {Air }} c_{p, \text { Air }}}{c_{v, \text { Air }} V_{\text {Inlet }}} & 0 & 0 \\
0 & 1 & 0 \\
0 & 0 & 0 \\
0 & 0 & 1
\end{array}\right], \quad C=\left[\begin{array}{cccc}
1 & 0 & 0 & 0 \\
0 & 0 & 0 & \frac{R_{\mathrm{Exh}} T_{\mathrm{Exh}}^{\mathrm{moy}}}{V_{\mathrm{Exh}}}
\end{array}\right], \\
B_{\bar{w}}=\left[\begin{array}{llll}
0 & 0 & 0 & 0
\end{array}\right]^{T},
\end{gathered}
$$

where $T_{\mathrm{Exh}}^{\text {moy }}$ is the mean value of measurable variable $T_{\mathrm{Exh}}$.

The matrix $B_{f}$ and the vector $f_{a}$ are chosen as follows:

$$
\begin{gathered}
B_{f}=\left[\begin{array}{cr}
\frac{R_{\mathrm{Exh}} T_{\mathrm{EGR}} A_{\mathrm{EGRmax}} c_{p, \mathrm{Exh}}}{V_{\text {Inlet }} c_{v, \mathrm{Exh}}} & 0 \\
0 & 0 \\
A_{\mathrm{EGRmax}} & 0 \\
-A_{\mathrm{EGRmax}} & 1
\end{array}\right], \\
f_{a}=\left[\begin{array}{c}
\frac{P_{\mathrm{Exh}}}{\sqrt{R_{\mathrm{Exh}} T_{\mathrm{Exh}}}} \Psi_{\kappa_{\mathrm{Exh}}}\left(\frac{P_{\text {Inlet }}}{P_{\mathrm{Exh}}}\right) f_{\mathrm{EGR}}\left(u_{\mathrm{EGR}}\right) \\
\frac{P_{\mathrm{Exh}}}{\sqrt{T_{\mathrm{Exh}}}} \tau\left(\frac{P_{\mathrm{Exh}}}{P_{\mathrm{Atm}}}, u_{\mathrm{XVNT}}\right)
\end{array}\right] .
\end{gathered}
$$

Finally, the functions $g$ and $f$ are given by

$$
\begin{aligned}
& g=\left[\begin{array}{lll}
W_{\text {HFM }} T_{\text {CAC }} & W_{\text {HFM }} & W_{\text {Fuel }}
\end{array}\right]^{T}, \\
& f=\left[\begin{array}{c}
\frac{R_{\text {Inlet }} P_{\text {Inlet }}}{c_{v, \text { Inlet }}} \\
0 \\
\frac{A_{\mathrm{EGR}} m_{\mathrm{Exh}} \Psi_{\kappa_{\mathrm{Exh}}} T_{\mathrm{Exh}}}{\sqrt{R_{\mathrm{Exh}} T_{\mathrm{Exh}}}} \\
-\frac{R_{\mathrm{Exh}} T_{\mathrm{Exh}} m_{\mathrm{Exh}} \tau\left(P_{\mathrm{Exh}} / P_{\mathrm{Atm}}, u_{\mathrm{XVNT}}\right)}{V_{\mathrm{Exh}} \sqrt{T_{\mathrm{Exh}}}}
\end{array}\right] .
\end{aligned}
$$


As mentioned in Section 3, the parameters $f_{\mathrm{vol}}, f_{\mathrm{EGR}}$, $\tau$, and $h$ are computed by interpolation in lookup tables. However, in this work, we are interested in the diagnosis in the steady state. So, we choose these parameters constant at each operating point to validate our approach. Furthermore, the proposed approach can take easily the parameter variation into account. For example, if we choose $f_{\mathrm{vol}}$ as variable parameter, the variable $\rho$ can be chosen as $\rho=f_{\text {vol }} N_{\text {Eng }}$ instead of $\rho=N_{\text {Eng }}$. By doing this, there is no need to change the design approach since the engine model has always the form (3a) and (3b).

\section{Conflict of Interests}

The authors declare that there is no conflict of interests regarding the publication of this paper.

\section{Acknowledgments}

This work was produced in the framework of SCODECE (Smart Control and Diagnosis for Economic and Clean Engine), a European territorial cooperation project partfunded by the European Regional Development Fund (ERDF) through the INTERREG IV A 2 Seas Programme, and the Research Department of the Region Nord Pas de Calais, France.

\section{References}

[1] CARB, "California's obd-ii regulation (section 1968.1 title 13, california code of regulations)," in Resolution 93-40, pp. 220.7(h)220.12(h), 1993.

[2] J. Gertler, M. Costin, X. Fang, Z. Kowalczuk, M. Kunwer, and R. Monajemy, "Model based diagnosis for automotive enginesalgorithm development and testing on a production vehicle," IEEE Transactions on Control Systems Technology, vol. 3, no. 1, pp. 61-68, 1995.

[3] M. Nyberg and A. Perkovic, "Model based diagnosis of leaks in the air-intake system of an siengine," SAE 980514, SAE International, 1998.

[4] M. Nyberg, "Model-based diagnosis of an automotive engine using several types of fault models," IEEE Transactions on Control Systems Technology, vol. 10, no. 5, pp. 679-689, 2002.

[5] M. Nyberg and T. Stutte, "Model based diagnosis of the air path of an automotive diesel engine," Control Engineering Practice, vol. 12, no. 5, pp. 513-525, 2004.

[6] I. Djemili, A. Aitouche, and V. Cocquempot, "Structural analysis for air path of an automotive diesel engine," in Proceedings of the International Conference on Communications, Computing and Control Applications (CCCA '11), pp. 1-6, Hammamet, Tunisia, March 2011.

[7] V. De Flaugergues, V. Cocquempot, M. Bayart, and N. Lefebvre, "Exhaustive search of residuals computation schemes using macro-graphs and invertibility constraints," in Proceedings of the 7th IFAC Symposium on Fault Detection, Supervision and Safety of Technical Processes, Barcelona, Spain, 2009.

[8] V. de Flaugergues, V. Cocquempot, M. Bayart, and M. Pengov, "On non-invertibilities for structural analysis," in Proceedings of the 21st International Workshop on Principles of Diagnosis (DX '10), Portland, Ore, USA, 2010.
[9] R. Ceccarelli, C. Canudas-de Wit, P. Moulin, and A. Sciarretta, "Model-based adaptive observers for intake leakage detection in diesel engines," in Proceedings of the IEEE American Control Conference (ACC '09), pp. 1128-1133, St. Louis, Mo, USA, June 2009.

[10] R. Ceccarelli, P. Moulin, and C. Canudas-de-Wit, "Robust strategy for intake leakage detection in Diesel engines," in Proceedings of the IEEE International Conference on Control Applications (CCA '09), pp. 340-345, IEEE, Saint Petersburg, Russia, July 2009.

[11] I. Djemili, A. Aitouche, and V. Cocquempot, "Adaptive observer for Intake leakage detection in diesel engines described by Takagi-Sugeno model," in Proceedings of the 19th Mediterranean Conference on Control \& Automation (MED '11), pp. 754-759, Corfu, Greece, June 2011.

[12] B. Boulkroune, I. Djemili, A. Aitouche, and V. Cocquempot, "Nonlinear unknown input observer design for diesel engines," in Proceedings of the IEEE American Control Conference (ACC '13), pp. 1076-1081, Washington, DC, USA, June 2013.

[13] E. Höckerdal, E. Frisk, and L. Eriksson, "EKF-based adaptation of look-up tables with an air mass-flow sensor application," Control Engineering Practice, vol. 19, no. 5, pp. 442-453, 2011.

[14] E. Höckerdal, E. Frisk, and L. Eriksson, "Observer design and model augmentation for bias compensation with a truck engine application," Control Engineering Practice, vol. 17, no. 3, pp. 408417, 2009.

[15] C. Svärd, M. Nyberg, E. Frisk, and M. Krysander, "Automotive engine FDI by application of an automated model-based and data-driven design methodology," Control Engineering Practice, vol. 21, no. 4, pp. 455-472, 2013.

[16] P. Kudva, N. Viswanadham, and A. Ramakrishna, "Observers for linear systems with unknown inputs," IEEE Transactions on Automatic Control, vol. AC-25, no. 1, pp. 113-115, 1980.

[17] M. Hou and P. C. Muller, "Design of observers for linear systems with unknown inputs," IEEE Transactions on Automatic Control, vol. 37, no. 6, pp. 871-875, 1992.

[18] W. Chen and M. Saif, "Unknown input observer design for a class of nonlinear systems: an lmi approach," in Proceedings of the IEEE American Control Conference, Minneapolis, Minn, USA, June 2006.

[19] M. Kao and J. J. Moskwa, "Turbocharged diesel engine modeling for nonlinear engine control and state estimation," Journal of Dynamic Systems, Measurement and Control, Transactions of the ASME, vol. 117, no. 1, pp. 20-30, 1995.

[20] J. Heywood, Internal Combustion Engine Fundamentals, McGraw-Hill Series in Mechanical Engineering, McGraw-Hill, New York, NY, USA, 1992.

[21] J. Wahlström and L. Eriksson, "Modelling diesel engines with a variable-geometry turbocharger and exhaust gas recirculation by optimization of model parameters for capturing non-linear system dynamics," Proceedings of the Institution of Mechanical Engineers, Part D: Journal of Automobile Engineering, vol. 225, no. 7, pp. 960-986, 2011.

[22] M. Witczak, Modelling and Estimation Strategies for Fault Diagnosis of Nonlinear Systems, Springer, Berlin, Germany, 2007.

[23] A. Zemouche and M. Boutayeb, "Sobolev norms-based state estimation and input recovery for a class of nonlinear systems design and experimental results," IEEE Transactions on Signal Processing, vol. 57, no. 3, pp. 1021-1029, 2009.

[24] G. Phanomchoeng, R. Rajamani, and D. Piyabongkarn, "Nonlinear observer for bounded Jacobian systems, with applications 
to automotive slip angle estimation," IEEE Transactions on Automatic Control, vol. 56, no. 5, pp. 1163-1170, 2011.

[25] B. Boulkroune, I. Djemili, A. Aitouche, and V. Cocquempot, "Robust nonlinear observer design for actuator fault detection in diesel engines," International Journal of Applied Mathematics and Computer Science, vol. 23, no. 3, pp. 557-569, 2013.

[26] M. Gálvez-Carrillo and M. Kinnaert, "Fault detection and isolation in current and voltage sensors of doubly-fed induction generators," in Proceedings of the 7th IFAC Symposium on Fault Detection, Supervision and Safety of Technical Processes (SafeProcess '09), Barcelona, Spain, 2009.

[27] I. Nikiforov, "A simple recursive algorithm for diagnosis of abrupt changes insignals and systems," in Proceedings of the IEEE American Control Conference, vol. 3, pp. 1938-1942, Philadelphia, Pa, USA, 1998.

[28] M. Kinnaert, D. Vrančić, E. Denolin, D. Juričić, and J. Petrovčić, "Model-based fault detection and isolation for a gas-liquid separation unit," Control Engineering Practice, vol. 8, no. 11, pp. 1273-1283, 2000. 


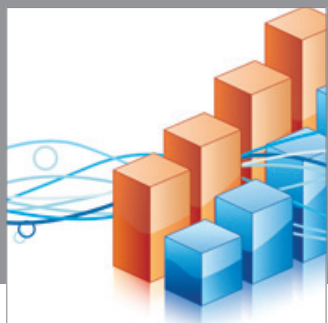

Advances in

Operations Research

mansans

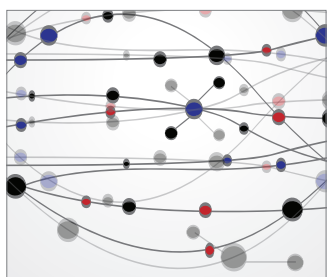

The Scientific World Journal
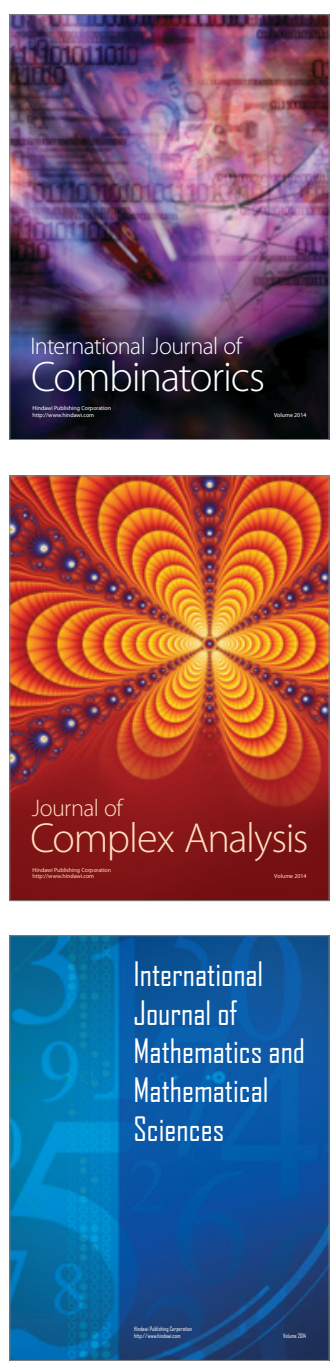
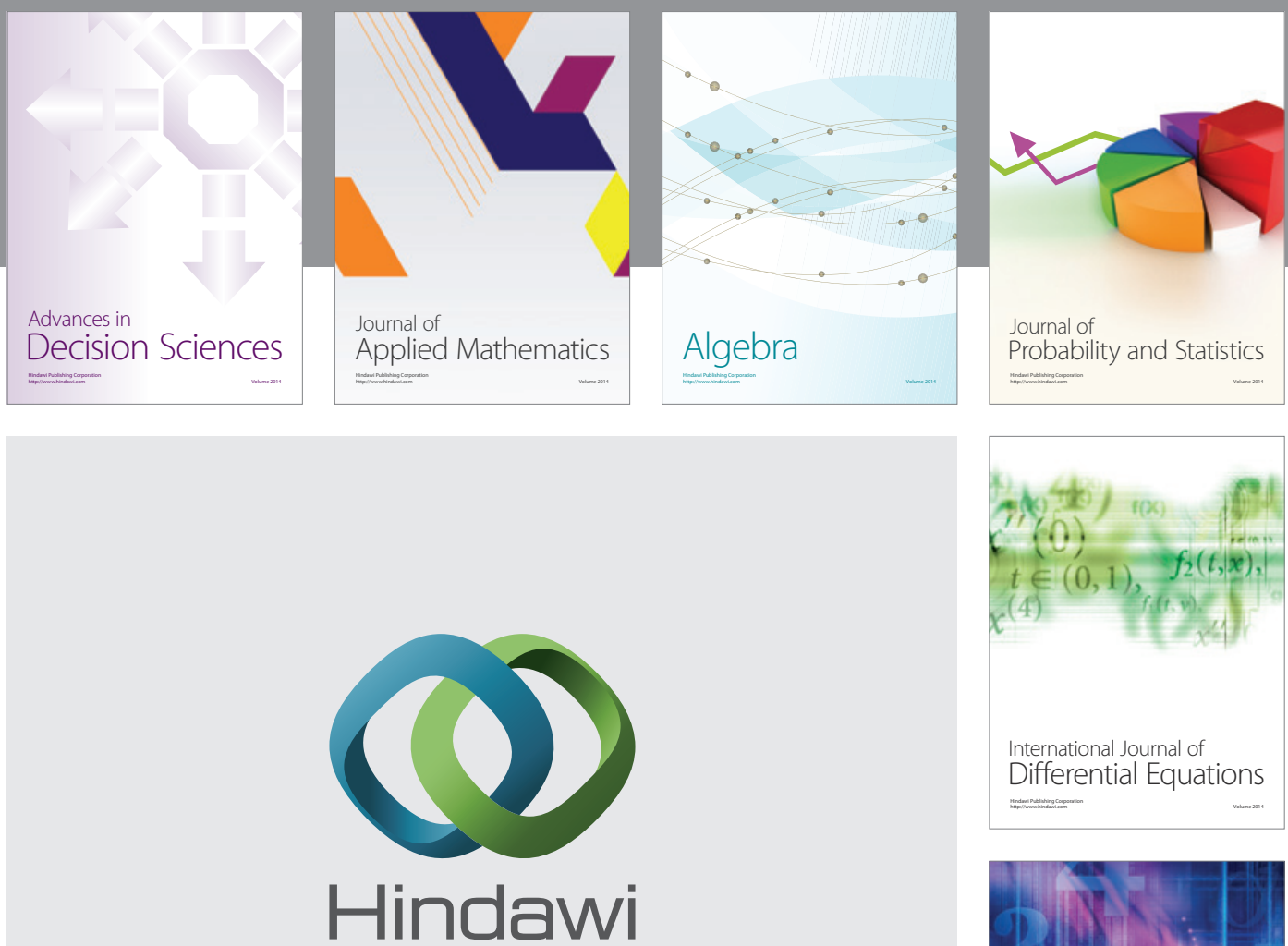

Submit your manuscripts at http://www.hindawi.com
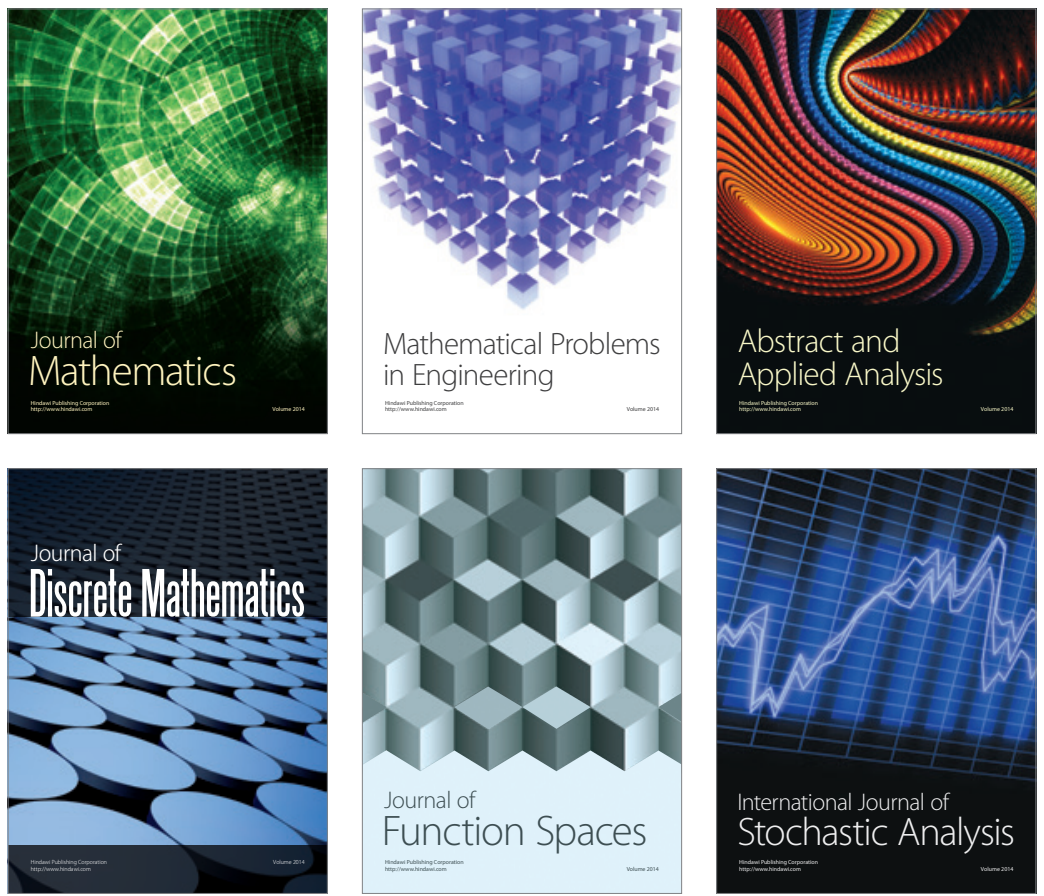

Journal of

Function Spaces

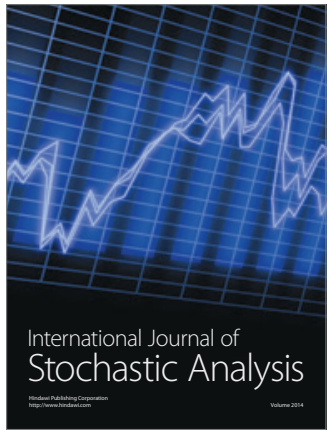

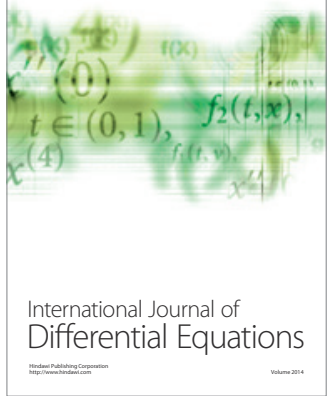
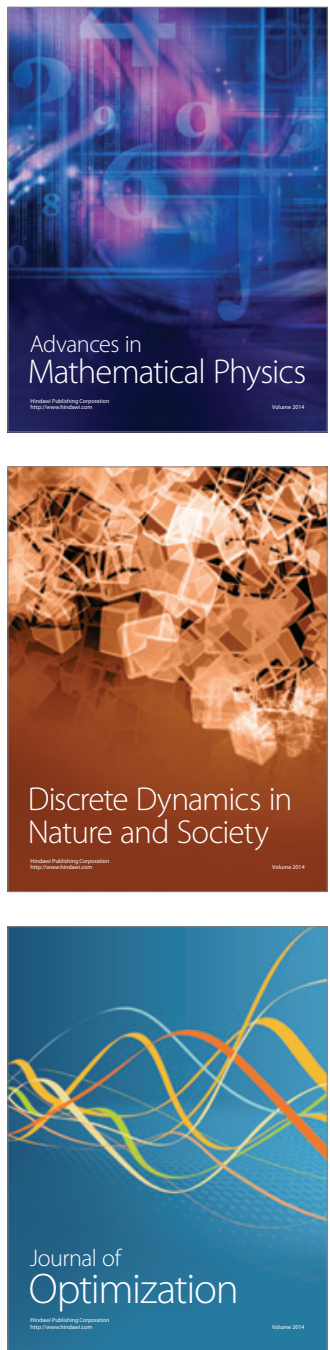